\title{
INSTABILITY OF PERIODIC TRAVELLING WAVES WITH MEAN ZERO FOR A 1D BOUSSINESQ SYSTEM*
}

\author{
JOSÉ R. QUINTERO ${ }^{\dagger}$ AND JUAN C. MUÑOZ
}

\begin{abstract}
We consider herein the instability properties of the periodic traveling wave solutions of a general nonlinear Boussinesq system related with a dispersive model for the 1D propagation of nonlinear long water waves with small amplitude, via an adaptation of the result of M. Grillakis, J. Shatah, and W. Strauss for systems with a special Hamiltonian structure. In a particular case of this general system, we use Jacobian elliptic functions to build a curve of $L$-periodic traveling wave solutions having mean zero in $[0, L]$ and also verify the validity of the criteria used to establish instability, in a specific range of the wave speed. Furthermore, we provide numerical evidence on a type of instability arising when perturbing with small amplitude disturbances by using a highlyaccurate spectral numerical scheme.
\end{abstract}

Key words. Dispersive equations, periodic traveling-waves, cnoidal, snoidal, waves, orbital instability.

AMS subject classifications. 76B25, 35Q51, 35Q53.

\section{Introduction}

In this paper we consider the study of orbital instability of periodic traveling wave solutions for the one-dimensional Boussinesq system for the 1D propagation of nonlinear dispersive long water waves with small amplitude

$$
\left\{\begin{array}{l}
q_{t}=r_{x} \\
r_{t}=B^{-1}\left(A q_{x}-n r q^{n-1} q_{x}-2 r_{x} q^{n}\right),
\end{array}\right.
$$

where $A=I-a \partial_{x}^{2}$ and $B=I-b \partial_{x}^{2}$ are linear operator defined through Fourier Series, $a$ and $b$ being positive numbers such that $a-b=\sigma-1 / 3\left(\sigma^{-1}\right.$ is known as the Bond number which is associated with surface tension). We also establish the existence of a curve of periodic traveling wave solutions for this system for $n=2$. This Boussinesq system is obtained from the rescaled version of the 1D generalized Benney-Luke model

$$
\Phi_{t t}-\Phi_{x x}+\mu\left(a \Phi_{x x x x}-b \Phi_{x x t t}\right)+\epsilon\left(n \Phi_{t}\left(\Phi_{x}\right)^{n-1} \Phi_{x x}+2\left(\Phi_{x}\right)^{n} \Phi_{x t}\right)=0,
$$

by using the variables $r=\Phi_{t}$ and $q=\Phi_{x}$, where the function $\Phi(x, t)$ represents the non-dimensional velocity potential at the bottom fluid boundary, $\mu$ represents the long-wave parameter (dispersion coefficient), $\epsilon$ represents the amplitude parameter (nonlinear parameter).

J. Quintero and R. Pego derived for $n=1$ the model (1.2) as a first order approximation (in one spatial dimension) to describe the evolution of long waves propagating on the surface of an ideal fluid under the force of gravity and taking into account the effect of surface tension (see [19]). It is important to note that Benney and Luke derived in [5] a similar model to (1.2) with $n=1$, but neglected surface tension effects. P. Milewski [13] also generalized this equation to include joint effects of topographical forcing, mean flow, and surface tension and Milewski et al. $[14,15,16]$ conducted a numerical study of several properties of solutions of Benney-Luke-type models in the two-dimensional case.

\footnotetext{
*Received: August 5, 2011; accepted (in revised form): February 26, 2012. Communicated by Paul Milewski.

${ }^{\dagger}$ Departamento de Matemáticas, Universidad del Valle, Colombia (quinthen@univalle.edu.co).

‡Departamento de Matemáticas, Universidad del Valle, Colombia (jcarlmz@yahoo.com).
} 
As we know, solitary waves have played an important role in the last decades in the study of dynamics of wave propagation in many applied models such as fluid dynamics, optics, acoustics, oceanography, and weather forecasting, among others. A recent important application is the use of optical solitons in fibers as an efficient (reliable and fast) means of long-distance communication [11]. This means that the study of these types of solutions and their properties such as existence, orbital stability/instability under small perturbations and other properties is of great interest and is capturing the attention of researchers from the analytical and numerical view point. Much of the effort has been focused on models for unidirectional wave propagation or with the full Euler equations for surface and internal waves. However less attention has been given to establish results pertaining to traveling wave solutions of systems of dispersive evolution equations under periodic spatial conditions.

The Boussinesq System (1.2) with nonlinear exponent $n$ is closely related to the generalized $\mathrm{KdV}$ equation which describes motions of long waves in shallow water under gravity and in an one-dimensional anharmonic nonlinear lattice in the case that $n=2$ [24]. The nonlinear generalized KdV equation is also an important mathematical model with several applications in quantum mechanics, nonlinear optics, and plasma physics, among others (see [25],[26] and references therein). Furthermore, we are interested in studying what effect dispersion and the exponent $n$ have on traveling-wave solutions of system (1.1) and their stability/instability behavior. These facts motivate our study of this system with generalized nonlinear exponent $n$ and in particular the case $n=2$.

The stability of traveling wave solutions for models related with the system considered in the paper as KdV type models and the Schrödinger equation has been widely studied. In [6], Bona et. al. gave sufficient and necessary conditions for stability of solitons for the GKdV. In [2], Albert et. al. gave sufficient conditions for stability of solitary-wave solutions of model equations for long waves. In [4], Angulo et. al. established stability of cnoidal traveling wave solutions for KdV model. In [3], Angulo proved stability of dnoidal traveling wave solutions for the GKdV. In [20], Quintero gave sufficient conditions for stability of periodic traveling waves having the mean zero property for the GKdV. Stability of periodic traveling wave solutions for the focusing Schrödinger equation were also treated recently in [3] and [8] by Angulo et. al. and Gallay et. al., respectively. Stability and instability analysis of solitary waves for a dispersive system of KdV-type equations was performed in [1] by Alarcon et. al. The problem of stability of periodic solutions of two coupled nonlinear Schrödinger equations was considered by Tan et. al. in [23] from the numerical view point and some stability conditions in terms of the parameters for the model are provided based on a linear analysis. Modulational instability of nonlinearly interacting two-dimensional waves in deep water modeled by a pair of two-dimensional coupled Schrödinger equations was treated in [22] by Shukla et. al. A spectral stability analysis for solitary waves in a system of coupled nonlinear Schrödinger equations is developed in [18] by Pelinovsky et. al. Finally, Quintero et. al., in [21], established orbital stability of cnoidal waves for the 1D Boussinesq equation for $n=1$ when wave speed is small enough. Recent developments regarding the stability of traveling wave solutions of dispersive models such as the KdV equation and some Boussinesq systems using the Krein signature, a reinterpretation of the works by M. Grillakis, J. Shatah, and W. Strauss, are [27, 28, 29, 30, 31, 32] and [33].

In this paper for $n=2$, we will build $L$-periodic traveling waves $\Psi_{c}$ where the first component $\psi_{c}$ is a snoidal function for $c \in(1, \sqrt{a / b})$, or cnoidal for $c \in(\sqrt{a / b}, 1)$. 
Adapting the work by Grillakis et. al. [9], we further establish orbital instability of snoidal type solutions of the Boussinesq system (1.1) in the case that $a>b>\frac{a}{9}, n=2$, and the wave speed satisfies $1<c<\min \left(c_{*}, \sqrt{\frac{1+\frac{4 \pi^{2} a}{L^{2}}}{1+\frac{4 \pi^{2} b}{L^{2}}}}\right)$, where $c_{*}$ is such that the function $d(c)=\mathcal{F}\left(\Psi_{c}\right)$ is concave for $1<c<c_{*}$. Here $\mathcal{F}$ denotes the action functional associated to the model and the constant $L>0$ denotes the fundamental period of the snoidal solution.

In the other range of the wave speed (i.e. $c \in(\sqrt{a / b}, 1)$ ), we see that results in [9] cannot be applied due to the failure of the spectral hypothesis on the second variation of the action functional around the traveling wave solution. Thus, in this case we use the spectral numerical scheme introduced in [17] for exploring the instability issue and get an insight on the nature of the instability mechanism involved in the phenomenon.

This paper is organized as follows. In Section 2, we adapt some results in [9] to prove instability of the periodic traveling wave, assuming the concavity of the function $d$ and the condition $\left\langle\mathcal{F}^{\prime \prime}\left(\Psi_{c}\right), \Psi_{c}\right\rangle<0$. We will see that the Boussinesq System (1.1) has a Hamiltonian structure in the variable $(q, p)$ ( $p$ is the conjugate variable of momentum), which facilitates the analysis of the spectral properties of the second variation of the action functional $\mathcal{F}$. We also discuss the well-posedness of the Cauchy problem associated to the Boussinesq System (1.1) in the canonical variables. In Section 3 , for $n=2$ we build periodic traveling waves of fundamental period $L$ using Jacobian elliptic functions. Depending on the wave speed we have that the traveling solution $\left(\psi_{c}, p_{c}\right)$ has $\psi_{c}$ of either snoidal or cnoidal type. We also prove the existence of a smooth curve of traveling wave solutions for (1.1) with a fixed period $L$. In Section 4, we see that the condition $\left\langle\mathcal{F}^{\prime \prime}\left(\Psi_{c}\right), \Psi_{c}\right\rangle<0$ holds for $1>\frac{b}{a}>\frac{n}{n+(n+2)^{2}}$ and wave speed $1<|c|<\sqrt{\frac{1+\frac{4 \pi^{2} a}{L^{2}}}{1+\frac{4 \pi^{2} b}{L^{2}}}}$, for $n$ even. For $n=2$, we verify the spectral conditions on the operator $\mathcal{F}^{\prime \prime}\left(\Psi_{c}\right)$ assumed in Section 2, which depends on the spectrum of the periodic eigenvalue problem associated with the linear operator

$$
\mathcal{L}_{s n}=-\left(a-b c^{2}\right) \frac{d^{2}}{d x^{2}}+\left(1-c^{2}+4 c \psi^{2}\right)
$$

where $\psi$ is a snoidal type function, in case $1<c<\sqrt{a / b}$. We establish the concavity of $d$ and also the instability result, for wave speed $1<c \ll \sqrt{a / b}$. In Section 5 , we describe the numerical experiments performed for the two families of periodic traveling-wave solutions with the mean zero property studied in the paper for $n=2$.

\section{Instability criteria via the Grillakis-Shatah-Strauss approach}

In this section we will adapt [9] to provide a criterion of instability for periodic traveling waves $\Psi_{c}$ for the Boussinesq System (1.1), when $\Psi_{c}$ has its first component with the mean zero property.

2.1. Hamiltonian Structure. Hereafter we will set the functional space $X=\mathcal{W}_{L}^{1} \times H_{\text {per }}^{1}([0, L])$, where $\mathcal{W}_{L}^{s}$ is the mean zero space of $L$-periodic functions in $[0, L]$ defined as

$$
\mathcal{W}_{L}^{s}=\left\{f \in H_{\text {per }}^{s}([0, L]): \int_{0}^{L} f(x) d x=0\right\}, \quad s \in \mathbb{Z} .
$$


We note that $f \in \mathcal{W}_{L}^{s}$ if and only if $f(x)=\sum_{-\infty}^{\infty} \hat{f}_{n} e^{2 \pi i n x / L}$ with $\hat{f}_{0}=0$, and

$$
\sum_{-\infty}^{\infty}\left(1+\frac{4 \pi^{2}|n|^{2}}{L^{2}}\right)^{s}\left|f_{n}\right|^{2}<\infty
$$

Note that (1.1) can be written as the system

$$
\begin{aligned}
q_{t} & =r_{x}, \\
{\left[B r+\frac{1}{n+1} q^{n+1}\right]_{t} } & =\partial_{x}\left[A q-r q^{n}\right],
\end{aligned}
$$

where $A=I-a \partial_{x}^{2}$ and $B=I-b \partial_{x}^{2}$ are defined through Fourier series. Note that if we introduce the conjugate momentum variable $p=B r+\frac{1}{n+1} q^{n+1}$, then the previous system becomes formally

$$
\begin{aligned}
q_{t} & =r_{x}, \\
p_{t} & =\partial_{x}\left[A q-r q^{n}\right] .
\end{aligned}
$$

Via these canonical variables, we find the Hamiltonian structure $\mathcal{H}: X \rightarrow \mathbb{R}$ for (1.1) is given by

$$
\mathcal{H}=\frac{1}{2} \int_{0}^{L}\left(p-\frac{1}{n+1} q^{n+1}\right) B^{-1}\left(p-\frac{1}{n+1} q^{n+1}\right)+q A q d x .
$$

We find that $\mathcal{H}^{\prime}: X \rightarrow X^{*}$ is given by

$$
\mathcal{H}^{\prime}\left(\begin{array}{l}
q \\
p
\end{array}\right)=\left(\begin{array}{c}
-B^{-1}\left(p-\frac{1}{n+1} q^{n+1}\right) q^{n}+A q \\
B^{-1}\left(p-\frac{1}{n+1} q^{n+1}\right)
\end{array}\right) .
$$

Note that

$$
\begin{aligned}
& q_{t}=\partial_{x} B^{-1}\left(p-\frac{1}{n+1} q^{n+1}\right)=\partial_{x} \mathcal{H}_{p}, \\
& p_{t}=\partial_{x}\left(-B^{-1}\left(p-\frac{1}{n+1} q^{n+1}\right) q^{n}+A q\right)=\partial_{x} \mathcal{H}_{q} .
\end{aligned}
$$

This implies that system (1.1) is equivalent to the system which is in canonical Hamiltonian form

$$
\begin{aligned}
\left(\begin{array}{l}
q \\
p
\end{array}\right)_{t} & =\partial_{x} \mathcal{J}_{0} \mathcal{H}^{\prime}\left(\begin{array}{l}
q \\
p
\end{array}\right) \\
& =\partial_{1} \mathcal{H}^{\prime}\left(\begin{array}{l}
q \\
p
\end{array}\right),
\end{aligned}
$$

where $\mathcal{J}_{1}=\partial_{x} \mathcal{J}_{0}$ and $\mathcal{J}_{0}$ is the linear operator given by

$$
\partial_{0}=\left(\begin{array}{ll}
0 & I \\
I & 0
\end{array}\right) .
$$

It is straightforward to see that the operator $\partial_{x}: \mathcal{W}_{L}^{s} \rightarrow \mathcal{W}_{L}^{s-1}$ is a bijection and that the operator $\partial_{x}: H_{\text {per }}^{s}([0, L]) \rightarrow H_{\text {per }}^{s-1}([0, L])$ is one to one (but not onto). This implies 
that the skew symmetric operator $\partial_{x} \mathcal{J}_{0}: \mathcal{W}_{L}^{0} \times H_{p e r}^{0}([0, L]) \subset X^{*} \rightarrow X$ is a one to one, but not onto. In other words, $\mathcal{J}_{1}$ does not satisfy the general framework in [9], which is also the case for solitons of the GKdV equation (see Bona et. al. [6]).

We also have that due to the translation invariance of the equation of motion, Noether's Theorem assures the existence of the following conserved quantity (charge),

$$
N\left(\begin{array}{l}
q \\
p
\end{array}\right)=\int_{0}^{L} p q d x
$$

In this canonical variables, we have the existence of a mass invariant functional

$$
\mathcal{J}(U)=\int_{0}^{L} U(x) d x
$$

which is cleverly utilized in the instability result in the case of the KdV type models (see $[6]$ ).

If we consider that $(q, p)(x, t)=(Q, P)(x-c t, t)$ is a solution of the equation (2.3), then we obtain that $(Q, P)$ has to satisfy the new system

$$
\left(\begin{array}{l}
Q \\
P
\end{array}\right)_{t}=\partial_{x} \partial_{0}\left[\mathcal{H}^{\prime}+c N^{\prime}\right]\left(\begin{array}{l}
Q \\
P
\end{array}\right)=\mathcal{J}_{1} \mathcal{F}^{\prime}\left(\begin{array}{l}
Q \\
P
\end{array}\right)
$$

where $\mathcal{F}=\mathcal{H}+c N$, showing that traveling waves are equilibrium solutions of system (2.4), up to constants. In accordance with the adaptation of the results in [9], we must analyze the spectrum of the operator (evaluated in $q=q_{c}$ and $p=p_{c}$ )

$$
\mathcal{F}^{\prime \prime}\left(\begin{array}{l}
q \\
p
\end{array}\right)=\left(\begin{array}{cc}
-n B^{-1}\left(p-\frac{1}{n+1} q^{n+1}\right) q^{n-1}+B^{-1}\left(q^{n}(\cdot)\right) q^{n}+A & -q^{n} B^{-1}+c I \\
-B^{-1}\left(q^{n}(\cdot)\right)+c I & B^{-1}
\end{array}\right) .
$$

If we want to find periodic traveling-waves solutions $(q, r)(x, t)=\left(q_{c}(x-c t), r_{c}(x-c t)\right)$ for system (1.1), we see that the couple $\left(q_{c}, p_{c}\right)$ satisfies the nonlinear system

$$
\begin{aligned}
& -c \partial_{x} q_{c}=\partial_{x} B^{-1}\left(p_{c}-\frac{1}{n+1} q_{c}^{n+1}\right) \\
& -c \partial_{x} p_{c}=\partial_{x}\left(B^{-1}\left(p_{c}-\frac{1}{n+1} q_{c}^{n+1}\right) q_{c}^{n}+A q_{c}\right) .
\end{aligned}
$$

Moreover, after integrating we have that

$$
\begin{aligned}
& -c q_{c}=B^{-1}\left(p_{c}-\frac{1}{n+1} q_{c}^{n+1}\right)+A_{0}, \\
& -c p_{c}=-B^{-1}\left(p_{c}-\frac{1}{n+1} q_{c}^{n+1}\right) q_{c}^{n}+A q_{c}+A_{1},
\end{aligned}
$$

where $A_{0}$ and $A_{1}$ are integration constants. Then we conclude that

$$
\begin{aligned}
p_{c} & =\frac{1}{n+1} q_{c}^{n+1}-c B q_{c}-A_{0}, \\
A q_{c}-c^{2} B q_{c}+\frac{(n+2) c}{n+1} q_{c}^{n+1} & =-A_{0} q_{c}^{n}-A_{1}+c A_{0} .
\end{aligned}
$$


On the other hand, if we look for periodic traveling-wave solutions $\Phi(x, t)=\phi(x-c t)$ for $(1.2)(\mu=\epsilon=1)$, we have that $q_{c}=\phi^{\prime}$ has mean zero and satisfies

$$
A \rho-c^{2} B \rho+\frac{(n+2) c}{n+1} \rho^{n+1}=\mathcal{A},
$$

where $\mathcal{A}$ is an integration constant. It follows by comparing the last two equations for $q_{c}$ that $A_{0}$ must be zero. In other words, the couple $\left(q_{c}, p_{c}\right)$ satisfies the nonlinear system

$$
\begin{aligned}
p_{c}-\frac{1}{n+1} q_{c}^{n+1}+c B q_{c} & =0, \\
A q_{c}-c^{2} B q_{c}+\frac{(n+2) c}{n+1} q_{c}^{n+1} & =-A_{1} .
\end{aligned}
$$

Note that if $q_{c}$ is a periodic solution with mean zero on $[0, L]$, then $A_{1} \neq 0$ for $n$ odd. This simple observation shows that $\Psi_{c}=\left(q_{c}, p_{c}\right)^{t}$ cannot be a critical point of the action functional, so we need to adapt the approach used in [9] to obtain orbital instability to the present case considered in this paper. We note that if $W \in X$, then

$$
\mathcal{F}^{\prime}\left(\Psi_{c}\right) W=\left\langle\left(\begin{array}{c}
A_{1} \\
0
\end{array}\right), W\right\rangle=0 .
$$

As we mention in the introduction, Grillakis et. al. in [9] established a general result which characterizes orbital instability for a general class of systems with a Hamiltonian structure of the form

$$
\partial_{t} V=\mathcal{J} E^{\prime}(V),
$$

where $\mathcal{J}$ is a skew operator on a Hilbert space. Now, in the periodic case in $[0, L]$, the model does not fit completely into the results in [9] since the eigenfunction $\chi_{c}$ associated with unique negative eigenvalue for $\mathcal{L}_{c, n}=\mathcal{F}^{\prime \prime}\left(q_{c}, p_{c}\right)$ does not necessarily have the mean zero property in $[0, L]$, and also because the operator $\mathcal{J}_{1}=\partial_{x} \mathcal{J}_{0}$ is not onto in $X$. So, we need to overcome this by extending and adapting slightly [9] to the periodic case, in spaces having the mean zero property in the first component.

2.2. Adaptation of the Grillakis-Shatah-Strauss approach. Hereafter we identify $\left(q_{c}, p_{c}\right)^{t}=\Psi_{c}$. Note that we have that $\partial_{x} \Psi_{c} \in\left(\mathcal{W}_{L}^{1}\right)^{2}$ is the eigenfunction of $\mathcal{L}_{c, n}$ associated with the zero eigenvalue. By hypothesis (H.2), we are assuming that $\lambda=0$ is a simple eigenvalue for $\mathcal{L}_{c, n}$, and the rest of the spectrum of $\mathcal{L}_{c, n}$ in $\left(H_{\text {per }}^{2}\right)^{2}$ is positive and bounded away from zero. Unfortunately, we do not know if the eigenfunction $\chi_{c}$ associated with the unique negative eigenvalue of $\mathcal{L}_{c, n}$ is in the space $X=\mathcal{W}_{L}^{1} \times H_{\text {per }}^{1}$.

In order to adapt [9] to establish instability of periodic traveling waves for the Boussinesq System (1.1), we will impose the same type of hypotheses adopted by Bona et. al. in [6] to obtain sufficient conditions to guarantee instability of $L$-periodic traveling wave solutions of the Boussinesq type system (1.1) in the space $X$, which satisfy that their first components have the mean zero property in $[0, L]$.

Assumptions on $\mathcal{L}_{c, n}$ and $\Psi_{c}$.

(H1) There is an interval $\left(c_{1}, c_{2}\right) \subset[1, \infty)$ such that for every $c \in\left(c_{1}, c_{2}\right)$, there is a solution $\Psi_{c}$ of (2.5). The curve $c \rightarrow \Psi_{c}$ is $C^{1}$ with values in $X$. 
(H2) The operator $\mathcal{L}_{c, n}$ has a unique, negative, simple eigenvalue with eigenfunction $\chi_{c} \in\left(H_{p e r}^{1}([0, L])\right)^{2}, \lambda=0$ is a simple eigenvalue of $\mathcal{L}_{c, n}$ with eigenfunction $\partial_{x} \Psi_{c}$, and the rest of the spectrum of $\mathcal{L}_{c, n}$ is positive and bounded away from zero. Moreover, the curve $c \rightarrow \chi_{c}$ is a continuous function with values in $\left(H_{p e r}^{1}([0, L])\right)^{2}$.

(H3) $\left\langle\mathcal{L}_{c, n}\left(\Psi_{c}\right), \Psi_{c}\right\rangle<0$ in some neighborhood of $c$.

Under these hypotheses, we are able to establish an important property of $\chi_{c}$ and $\Psi_{c}$.

Lemma 2.1. Assume that $\mathcal{L}_{c, n}$ satisfies condition $(\mathbf{H 2})$ and $\mathbf{( H 3 ) . ~ T h e n ~}\left\langle\chi_{c}, \Psi_{c}\right\rangle \neq 0$. In particular, $\chi_{c}$ can be taken such that $\left\langle\Psi_{c}, \chi_{c}\right\rangle>0$.

Proof. By the hypothesis, $\mathcal{L}_{c, n}$ has a unique, negative, simple eigenvalue, with eigenfunction $\chi_{c} \in\left(H_{p e r}^{1}([0, L])^{2}\right.$. Now note that

$$
\left\langle\Psi_{c}, \partial_{x} \Psi_{c}\right\rangle=1 / 2 \int_{0}^{L} \partial_{x}\left\|\Psi_{c}\right\|^{2} d x=0
$$

Then we can write $\Psi_{c}=\gamma \chi_{c}+\rho P_{0}$, for some $P_{0}$ in the positive subspace of $L^{2} \times L^{2}$ orthogonal to $\chi_{c}$ and $\partial_{x} \Psi_{c}$. In this case, $\left\langle\Psi_{c}, \chi_{c}\right\rangle=\gamma \neq 0$, otherwise $\Psi_{c}=\rho p_{0}$ and $\left\langle\mathcal{L}_{c, n}\left(\Psi_{c}\right), \Psi_{c}\right\rangle=\rho^{2}\left\langle\mathcal{L}_{c, n}\left(P_{0}\right), P_{0}\right\rangle \geq 0$, contradicting (H3).

Before we go further, we note that $d(c)=\mathcal{F}\left(\Psi_{c}\right)$. Then using that $\Psi_{c} \in X$, a direct computation shows that

$$
d^{\prime \prime}(c)=\frac{d}{d c} N\left(\Psi_{c}\right)
$$

Theorem 2.2. Under the hypotheses $(\mathbf{H 1})-(\mathbf{H 3})$, if $d^{\prime \prime}(c)<0$ then there is a curve $\omega \rightarrow \Phi_{\omega} \in X$ which passes through $\Psi_{c}$ and lies on the surface $N(U)=N\left(\Psi_{c}\right)$. Moreover, $\mathcal{H}(U)$ has a strict local maximum on the curve at $U=\Psi_{c}$ in $X$.

Proof. We proceed as in the work by Bona et. al. in [6]. For $\omega$ near $c$, we want to define a curve $(\omega, s) \rightarrow \Phi_{(\omega, s)} \in X$ of the form $\Phi_{\omega, s}=\Psi_{\omega}+s \Psi_{c}$, with $s(\omega)$ satisfying the conditions $s(c)=0$. We set the curve $\omega \rightarrow \Phi_{\omega}=\Phi_{(\omega, s(\omega))}$. Note for $s=0$ and $\omega=c$ that $\Phi_{c}=\Psi_{c}$ and $N\left(\Phi_{c}\right)=N\left(\Psi_{c}\right)$. We observe that

$$
\begin{aligned}
\left.\frac{\partial}{\partial_{s}} N\left(\Phi_{\omega}\right)\right|_{\{s=0, \omega=c\}} & =N^{\prime}\left(\Psi_{c}\right)\left(\Psi_{c}\right) \\
& =\int_{0}^{L}\left\langle\left(\begin{array}{c}
p_{c} \\
q_{c}
\end{array}\right),\left(\begin{array}{c}
q_{c} \\
p_{c}
\end{array}\right)\right\rangle d x \\
& =2 \int_{0}^{L} p_{c} q_{c} d x \\
& =-\frac{2}{c(n+2)} \int_{0}^{L}\left(c^{2}(n+1) q_{c} B q_{c}+q_{c} A q_{c}\right) d x<0
\end{aligned}
$$

since $A$ and $B$ are positive operators. From the Implicit Function Theorem, we assure the existence of a function $s(\omega)$ defined for $\omega$ near $c$ such that $N\left(\Phi_{\omega}\right)$ is constant along the curve $s(\omega)$. Now, since $\Psi_{\omega} \in X$ we have that

$$
\left.\partial_{\omega} \mathcal{H}\left(\Phi_{\omega}\right)\right|_{\{\omega=c\}}=\left\langle\mathcal{H}^{\prime}\left(\Phi_{c}\right),\left.\frac{d \Phi_{\omega}}{d \omega}\right|_{\{\omega=c\}}\right\rangle=0
$$


Moreover, since $N\left(\Phi_{\omega}\right)=N\left(\Psi_{c}\right)$, we conclude that

$$
\begin{aligned}
\frac{d^{2}}{d \omega^{2}} \mathcal{H}\left(\Phi_{\omega}\right) & =\frac{d^{2}}{d \omega^{2}}\left(\mathcal{H}\left(\Phi_{\omega}\right)+\omega N\left(\Phi_{\omega}\right)\right) \\
& =\left\langle\mathcal{H}^{\prime}\left(\Phi_{\omega}\right)+\omega N^{\prime}\left(\Phi_{\omega}\right), \frac{d^{2} \Phi_{\omega}}{d \omega^{2}}\right\rangle+\left\langle\left(\mathcal{H}^{\prime \prime}\left(\Phi_{\omega}\right)+\omega N^{\prime \prime}\left(\Phi_{\omega}\right)\right)\left(\frac{d \Phi_{\omega}}{d \omega}\right), \frac{d \Phi_{\omega}}{d \omega}\right\rangle .
\end{aligned}
$$

Evaluating this at $\omega=c$, and after noting that

$$
\left.\mathcal{A} \frac{d^{2}}{d \omega^{2}} \int_{0}^{L} \Phi_{\omega}(x)\right|_{\{\omega=c\}} d x=0
$$

we have that

$$
\left.\frac{d^{2}}{d \omega^{2}} \mathcal{H}\left(\Phi_{\omega}\right)\right|_{\{\omega=c\}}=\left\langle\mathcal{L}_{c, n} Y, Y\right\rangle, \quad \text { where } \quad Y=\left.\frac{d \Phi_{\omega}}{d \omega}\right|_{\{\omega=c\}}=\frac{d \Psi_{c}}{d c}+s^{\prime}(c) \Psi_{c} \in X .
$$

From the fact that $N$ is constant along $\Psi_{\omega}$, we have from equation (2.6) that

$$
\begin{aligned}
0=\left.\frac{d}{d \omega} N\left(\Phi_{\omega}\right)\right|_{\{\omega=c\}} & =\int_{0}^{L} N^{\prime}\left(\Psi_{c}\right) Y d x \\
& =\left\langle N^{\prime}\left(\Psi_{c}\right), \frac{d}{d c} \Psi_{c}\right\rangle+s^{\prime}(c)\left\langle N^{\prime}\left(\Psi_{c}\right), \Psi_{c}\right\rangle \\
& =\frac{d}{d c} N\left(\Psi_{c}\right)+s^{\prime}(c)\left\langle N^{\prime}\left(\Psi_{c}\right), \Psi_{c}\right\rangle \\
& =d^{\prime \prime}(c)+s^{\prime}(c)\left\langle N^{\prime}\left(\Psi_{c}\right), \Psi_{c}\right\rangle
\end{aligned}
$$

Thus we conclude that

$$
\int_{0}^{L} N^{\prime}\left(\Psi_{c}\right) Y d x=\left\langle\partial_{0}\left(\Psi_{c}\right), \Psi_{c}\right\rangle=0, \quad \text { and } \quad d^{\prime \prime}(c)=-s^{\prime}(c)\left\langle N^{\prime}\left(\Psi_{c}\right), \Psi_{c}\right\rangle .
$$

On the other hand,

$$
\mathcal{L}_{c, n}\left(\frac{d \Psi_{c}}{d c}\right)=\left(\begin{array}{c}
\partial_{c} \mathcal{A} \\
0
\end{array}\right)-N^{\prime}\left(\Psi_{c}\right)
$$

Using this, we have that

$$
\mathcal{L}_{c, n} Y=\mathcal{L}_{c, n}\left(\frac{d \Psi_{c}}{d c}\right)+s^{\prime}(c) \mathcal{L}_{c, n}\left(\Psi_{c}\right)=\left(\begin{array}{c}
\partial_{c} \mathcal{A} \\
0
\end{array}\right)-N^{\prime}\left(\Psi_{c}\right)+s^{\prime}(c) \mathcal{L}_{c, n}\left(\Psi_{c}\right)
$$

Since $\left\langle N^{\prime}\left(\Psi_{c}\right), Y\right\rangle=0$ and $\Psi_{c} \in X$, we conclude that

$$
\begin{aligned}
\left\langle\mathcal{L}_{c, n} Y, Y\right\rangle & =s^{\prime}(c)\left\langle\mathcal{L}_{c, n}\left(\Psi_{c}\right), Y\right\rangle \\
& =s^{\prime}(c)\left(\left\langle\mathcal{L}_{c, n}\left(\Psi_{c}\right), \frac{d}{d c} \Psi_{c}\right\rangle+s^{\prime}(c)\left\langle\mathcal{L}_{c, n}\left(\Psi_{c}\right), \Psi_{c}\right\rangle\right) \\
& =s^{\prime}(c)\left(\left\langle\mathcal{L}_{c, n}\left(\frac{d}{d c} \Psi_{c}\right), \Psi_{c}\right\rangle+s^{\prime}(c)\left\langle\mathcal{L}_{c, n}\left(\Psi_{c}\right), \Psi_{c}\right\rangle\right) \\
& =s^{\prime}(c)\left\langle\left(\begin{array}{c}
\partial_{c} \mathcal{A} \\
0
\end{array}\right)-N^{\prime}\left(\Psi_{c}\right), \Psi_{c}\right\rangle+\left(s^{\prime}(c)\right)^{2}\left\langle\mathcal{L}_{c, n}\left(\Psi_{c}\right), \Psi_{c}\right\rangle
\end{aligned}
$$




$$
\begin{aligned}
& =-s^{\prime}(c)\left\langle N^{\prime}\left(\Psi_{c}\right), \Psi_{c}\right\rangle+\left(s^{\prime}(c)\right)^{2}\left\langle\mathcal{L}_{c, n}\left(\Psi_{c}\right), \Psi_{c}\right\rangle \\
& =d^{\prime \prime}(c)+\left(s^{\prime}(c)\right)^{2}\left\langle\mathcal{L}_{c, n}\left(\Psi_{c}\right), \Psi_{c}\right\rangle<0,
\end{aligned}
$$

since we are assuming that $d^{\prime \prime}(c)<0$ and $\left\langle\mathcal{L}_{c, n}\left(\Psi_{c}\right), \Psi_{c}\right\rangle<0$. In other words, we have shown that

$$
\left.\frac{d^{2}}{d \omega^{2}} \mathcal{H}\left(\Phi_{\omega}\right)\right|_{\{\omega=c\}}=\left\langle\mathcal{L}_{c, n}(Y), Y\right\rangle<0
$$

as claimed.

Before we consider instability, we will prove an important result in this work,

Lemma 2.3. Under the hypotheses (H1)-(H3), we have that

$$
\left\langle Y, \chi_{c}\right\rangle \neq 0 .
$$

Proof. From previous result, we have that

$$
\left\langle\mathcal{L}_{c, n} Y, Y\right\rangle<0 .
$$

We note that $Y$ can be decomposed in $L^{2} \times L^{2}$ as $Y=\alpha_{0} \chi_{c}+\alpha_{1} \partial_{x} \Psi_{c}+\alpha_{2} P$, where $P$ belongs to the positive subspace of $\mathcal{L}_{c, n}$. Then a direct computation shows that

$$
\left\langle\mathcal{L}_{c, n} Y, Y\right\rangle=\lambda_{0} \alpha_{0}^{2}\left\langle\chi_{c}, \chi_{c}\right\rangle+\alpha_{2}^{2}\left\langle\mathcal{L}_{c, n} P, P\right\rangle<0 .
$$

This fact implies that $\alpha_{0}=\left\langle\chi_{c}, Y\right\rangle \neq 0$, since $\left\langle\mathcal{L}_{c, n} P, P\right\rangle \geq 0$ and $\lambda_{0}<0$.

Hereafter we only present the analogous result for the periodic case obtained in [9] (or in [6]), pointing out the appropriate changes. For $\epsilon>0$, consider the tube in $X$

$$
\mathcal{U}_{\epsilon}=\left\{V \in X: \inf _{r}\left\|V-\tau_{r} \Psi_{c}\right\|_{\left(H_{p e r}^{1}([0, L])\right)^{2}}<\epsilon\right\},
$$

where $\tau_{r}(f)(x)=f(r+x), x \in \mathbb{R}$. The set $\mathcal{U}_{\epsilon}$ is a neighborhood in $X$ of the collection of all translates of $\Psi_{c}$.

Lemma 2.4. There exist $\epsilon>0$ and a $C^{1}$ map $\alpha: \mathcal{U}_{\epsilon} \rightarrow \mathbb{R}$ such that

1. $\left\langle U\left(\cdot+\alpha(U), \partial_{x} \Psi_{c}\right\rangle=0\right.$,

2. $\alpha(U(\cdot+r))=\alpha(U)-r$ modulo the period,

3. $\alpha\left(\Psi_{c}\right)=0$,

4. $\alpha^{\prime}(U)=\frac{\partial_{x} \Psi_{c}(\cdot-\alpha(U))}{\int_{\mathbb{R}} U(x) \partial_{x}^{2} \Psi_{c}(x-\alpha(U)) d x}$.

Recall that we showed the existence of $Y=\frac{d \Psi_{c}}{d c}+s^{\prime}(c) \Psi_{c} \in X$ such that

$$
\left\langle\mathcal{L}_{c, n}(Y), Y\right\rangle<0 \quad \text { and } \quad\left\langle\mathcal{J}_{0}\left(\Psi_{c}\right), Y\right\rangle=0 .
$$

TheOREM 2.5. Under the hypotheses $(\mathbf{H 1})-(\mathbf{H 3})$, the function $\mathcal{B}: \mathfrak{U}_{\epsilon} \rightarrow X$ defined as

$$
\mathcal{B}(U)=Y(\cdot-\alpha(U))-\frac{\left\langle\partial_{0}(U), Y(\cdot-\alpha(U))\right\rangle}{\left\langle U, \partial_{x}^{2} \Psi_{c}(\cdot-\alpha(U))\right\rangle} \partial_{x}^{2} \partial_{0} \Psi_{c}(\cdot-\alpha(U))
$$

is $C^{1}$ from $\mathfrak{U}_{\epsilon}$ into $X$ such that it commutes with translations, $\mathcal{B}\left(\Psi_{c}\right)=Y$, and $\left\langle\mathcal{B}(U), \mathcal{J}_{0}(U)\right\rangle=0$, for $U \in \mathcal{U}_{\epsilon}$. 
The only comment is that $\mathcal{B}(U)$ has the mean zero property in $[0, L]$. In fact,

$$
\int_{0}^{L} \mathcal{B}(U)(x) d x=\int_{0}^{L} Y(x-\alpha(U)) d x-\left.\frac{\left\langle\mathcal{J}_{0}(U), Y(\cdot-\alpha(U))\right\rangle}{\left\langle U, \partial_{x}^{2} \Psi_{c}(\cdot-\alpha(U))\right\rangle} \partial_{x} \mathcal{J}_{0} \Psi_{c}(\cdot-\alpha(U))\right|_{0} ^{L}=0 .
$$

As in [9] (see also in [6]), consider the initial value problem

$$
\left\{\begin{aligned}
\frac{d U}{d \lambda} & =\mathcal{B}(U) \\
U(0) & =V \in \mathcal{U}_{\epsilon}
\end{aligned}\right.
$$

Corollary 2.6. Suppose the hypotheses (H1)-(H3) hold. If $U=R(\lambda, V)$ denotes the solution of the initial value problem (2.11), then we have

1. $R$ is a $C^{1}$ function for $|\lambda|<\lambda_{0}(v)$ for any $V \in \mathcal{U}_{\epsilon}$,

2. $R$ commutes with translations for each $\lambda$,

3. $N(R(\lambda, V))$ is independent of $\lambda$, and

4. $\frac{\partial R}{\partial \lambda}\left(0, \Psi_{c}\right)=Y$.

We want to point out that the proofs of the previous result and the the coming lemma are the same as those in [9] or [6].

Lemma 2.7. Under the hypotheses (H1)-(H3), there is a $C^{1}$ function $\Lambda:\left\{V \in \mathcal{U}_{\epsilon}\right.$ : $\left.N(V)=N\left(\Psi_{c}\right)\right\} \rightarrow \mathbb{R}$ such that

1. For all $V \in \mathcal{U}_{\epsilon}$ such that $V \notin\left\{\Psi_{c}(\cdot+s): s \in \mathbb{R}\right\}$,

$$
\mathcal{H}\left(\Psi_{c}\right)<\mathcal{H}(R(\Lambda(V), V)),
$$

2. For all $V \in \mathcal{U}_{\epsilon}$ such that $V \notin\left\{\Psi_{c}(\cdot+s): s \in \mathbb{R}\right\}$,

$$
\mathcal{H}\left(\Psi_{c}\right)<\mathcal{H}(V)+\Lambda(V)\left\langle\mathcal{H}^{\prime}(V), \mathcal{B}(V)\right\rangle .
$$

3. The curve $\Phi_{\omega}$ satisfies $\mathcal{H}\left(\Phi_{\omega}\right)<\mathcal{H}\left(\Psi_{c}\right)$ for $\omega \neq c, N\left(\Phi_{\omega}\right)=N\left(\Psi_{c}\right)$, and $\Lambda(V)\left\langle\mathcal{H}^{\prime}(V), \mathcal{B}(V)\right\rangle$ changes sign as $\omega$ passes through $c$.

Now we make precise the meaning of the stability and instability concept,

DeFinition 2.8. The periodic traveling wave $\Psi_{c}$ is stable if and only if for $\epsilon>0$ there exists $\eta>0$ such that if $V_{0} \in \mathcal{U}_{\eta}$, then $V(\cdot, t) \in \mathcal{U}_{\epsilon}$ for all $t \in[0, L]$, where $V(\cdot, t)$ denotes the unique solution of the Cauchy problem associated with the Boussinesq type system (1.1) in the space $X$, with initial condition $V(\cdot, 0)=V_{0}(\cdot)$. We will say that the periodic traveling wave $\Psi_{c}$ is unstable if $\Psi_{c}$ is not stable.

2.3. The Cauchy periodic problem. We note that system (1.1) has the form

$$
\left(\begin{array}{l}
q \\
r
\end{array}\right)_{t}=\mathcal{M}\left(\begin{array}{l}
q \\
r
\end{array}\right)+G\left(\begin{array}{l}
q \\
r
\end{array}\right)
$$

where

$$
\mathcal{M}=\left(\begin{array}{cc}
0 & \partial_{x} \\
\partial_{x} B^{-1} A & 0
\end{array}\right) \text { and } G\left(\begin{array}{l}
q \\
r
\end{array}\right)=\left(\begin{array}{c}
0 \\
-B^{-1}\left(2 r q^{n-1} q_{x}+2 q^{n} r_{x}\right)
\end{array}\right)
$$

As done by J. Quintero et. al. for $n=1$ in [21], it is easy to see that $\mathcal{M}$ is the infinitesimal generator of a bounded $C_{0}$-group $S(t)$ on $X^{1}=H_{p e r}^{1}([0, L]) \times H_{p e r}^{1}([0, L])$. Moreover, we have $\mathcal{M} \in \mathcal{L}_{b}\left(X^{2}, X^{1}\right)$, where $X^{2}=H_{\text {per }}^{2}([0, L]) \times \in H_{\text {per }}^{2}([0, L])$. Since $B^{-1}$ 
is a bounded linear operator from $L_{p e r}^{2}([0, L])$ to $H_{p e r}^{2}([0, L])$. On the other hand, if $f \in H_{\text {per }}^{1}([0, L])$, then $f \in L^{\infty}(\mathbb{R})$. Thus, if we assume that $(q, r)^{t} \in X^{1}$, then $q$ and $r$ are bounded functions. Since $q_{x}, r_{x} \in L_{p e r}^{2}([0, L])$, we conclude that $2 r q^{n-1} q_{x}+2 q^{n} r_{x} \in$ $L_{\text {per }}^{2}([0, L])$ and so we have that $B^{-1}\left(2 r q^{n-1} q_{x}+2 q^{n} r_{x}\right) \in H_{\text {per }}^{2}([0, L])$. In other words, we have shown that $G$ maps $X^{1}$ into $X^{2}$, meaning that $G$ gains some regularity. Moreover,

$$
\begin{aligned}
\left\|B^{-1}\left(2 r_{1} q_{1}^{n-1}\left(q_{1}\right)_{x}+2 q_{1}^{n}\left(r_{1}\right)_{x}\right)-B^{-1}\left(2 r_{2} q_{2}^{n-1}\left(q_{2}\right)_{x}+2 q_{2}^{n}\left(r_{2}\right)_{x}\right)\right\|_{H_{p e r}^{2}} \\
\leq\left\|\left(q_{1}, r_{1}\right)-\left(q_{2}, r_{2}\right)\right\|_{X^{1}},
\end{aligned}
$$

which means that $G$ is locally Lipschitz from $X^{1}$ into $X^{2}$. In other words, we have that

Theorem 2.9. Suppose that $\left(q_{0}, r_{0}\right)^{t} \in X^{1}$. Then the initial value problem associated with the system (2.12) with $\left(q_{0}, r_{0}\right)=(q(0, \cdot), r(0, \cdot))$ has a unique global mild solution $(q, r) \in X^{1}$. Moreover, if

$$
\left(q_{0}, r_{0}\right)^{t} \in Y_{1}=\left\{(q, r) \in\left(H_{p e r}^{1}([0, L])\right)^{2}: \int_{0}^{L} q(x) d x=0\right\},
$$

then the initial value problem associated with system (2.12) with $\left(q_{0}, r_{0}\right)=$ $(q(0, \cdot), r(0, \cdot))$ has a unique global mild solution $(q, r) \in Y_{1}$.

The result is a consequence that a solution $(q, r)$ satisfies the conserved quantities

$$
\mathcal{M}_{1}(q, r)=\int_{0}^{L} q(x) d x .
$$

Moreover, from this fact and the relation between $q, r$, and $p$, we are able to establish that

Corollary 2.10. Let $\left(q_{0}, p_{0}\right)^{t} \in X$. Then the initial value problem associated with system (2.3) with $\left(q_{0}, p_{0}\right)=(q(0, \cdot), p(0, \cdot))$ has a unique global mild solution $(q, p) \in X$.

2.4. Instability analysis. Following the setting used in Theorem 4.7 of [9], we define the operator $A$ in $\mathcal{U}_{\epsilon}$ as

$$
A(U)=-\langle Z(\cdot-\alpha(U)), U\rangle,
$$

where $\mathcal{J}_{1} Z=Y$. The first observation is that

$$
-\mathcal{J}_{1} A^{\prime}(U)=\mathcal{B}(U)
$$

In fact,

$$
A^{\prime}(U)=-Z(\cdot-\alpha(U))-\left\langle Z, \partial_{x} U(\cdot+\alpha(U))\right\rangle \alpha^{\prime}(U) .
$$

On the other hand, from (4) in Lemma (2.7),

$$
\partial_{1} \alpha^{\prime}(U)=\frac{\partial_{x}^{2} \partial_{0} \Psi_{c}(\cdot-\alpha(U))}{\int_{\mathbb{R}} U(x) \partial_{x}^{2} \Psi_{c}(x-\alpha(U)) d x} .
$$

Then we obtain that

$$
\left\langle Z, \partial_{x} U(\cdot+\alpha(U))\right\rangle=-\left\langle\mathcal{J}_{0} Y, U(\cdot+\alpha(U))\right\rangle=-\left\langle Y(\cdot-\alpha(U)), \mathcal{J}_{0} U\right\rangle .
$$


Putting the pieces together, we conclude that

$$
-\mathcal{J}_{1} A^{\prime}(U)=\mathcal{J}_{1} Z(\cdot-\alpha(U))-\langle Y(\cdot-\alpha(U)), K(U)\rangle \frac{\partial_{x}^{2} \partial_{0} \Psi_{c}(\cdot-\alpha(U))}{\int_{\mathbb{R}} U(x) \partial_{x}^{2} \Psi_{c}(x-\alpha(U)) d x}=\mathcal{B}(U) .
$$

TheOREM 2.11. Suppose the hypotheses (H1)-(H3) hold and that $d^{\prime \prime}(c)<0$. Then the $\Psi_{c}$-orbit is $X$-unstable with respect to the flow of equation (2.3).

Proof. Let $\Psi_{c}$ be fixed, and take $\epsilon>0$ small enough such that Lemma (2.7) holds. Thus, we can take $U_{0}=\Psi_{\omega}$ arbitrarily close to $\Psi_{c}$ such that

$$
N\left(\Psi_{\omega}\right)=N\left(\Psi_{c}\right), \quad \mathcal{H}\left(U_{0}\right)<\mathcal{H}\left(\Psi_{c}\right), \quad\left\langle\mathcal{H}^{\prime}\left(U_{0}\right), \mathcal{B}\left(U_{0}\right)\right\rangle>0 .
$$

Now, if $U(t)$ is the solution of the Boussinesq system with $U(0)=U_{0}$, then we have, as in Theorem 4.7 of [9], that

$$
\begin{aligned}
\frac{d}{d t} A(U(t)) & =\left\langle\frac{d}{d t} U(t), A^{\prime}(U(t))\right\rangle \\
& =\left\langle\mathcal{H}^{\prime}(U(t)),-\mathcal{J}_{1} A^{\prime}(U(t))\right\rangle \\
& =\left\langle\mathcal{H}^{\prime}(U(t)), \mathcal{B}(U(t))\right\rangle .
\end{aligned}
$$

Now in any interval $\left[0, t_{1}\right)$ on which $U(t) \in \mathcal{U}_{\epsilon}$,

$$
0<\mathcal{H}\left(\Psi_{c}\right)-\mathcal{H}\left(U_{0}\right)<\Lambda(U(t))\left\langle\mathcal{H}^{\prime}(U(t)), \mathcal{B}(U(t))\right\rangle<\left\langle\mathcal{H}^{\prime}(U(t)), \mathcal{B}(U(t))\right\rangle,
$$

where we are assuming that $\Lambda(U(t))<1$ for $\epsilon$ small enough, since $\Lambda\left(\Psi_{c}\right)=0$. As a consequence of this,

$$
\frac{d}{d t} A(U(t))>\mathcal{H}\left(\Psi_{c}\right)-\mathcal{H}\left(U_{0}\right)
$$

We note that for any $U \in \mathcal{U}_{\epsilon}$, we have the estimates

$$
\begin{aligned}
|A(U)| & \leq\|Z\|_{L_{p e r}^{2}}\left(\left\|\Psi_{c}\right\|_{H_{p e r}^{1}}+\epsilon\right) \\
& \leq\|Y\|_{L_{p e r}^{2}}\left(\left\|\Psi_{c}\right\|_{H_{p e r}^{1}}+\epsilon\right) .
\end{aligned}
$$

So, we are able to conclude from inequality (2.15) that

$$
\left(\mathcal{H}\left(\Psi_{c}\right)-\mathcal{H}\left(U_{0}\right)\right) t_{1} \leq C_{1}\|Y\|_{L_{p e r}^{2}}\left(\left\|\Psi_{c}\right\|_{H_{p e r}^{1}}+\epsilon\right) .
$$

But we are allowed to take $\|Y\|_{L_{p e r}^{2}}$ small (using $\rho Y$ instead $Y$ ) in such a way that

$$
t_{1} \leq \frac{C_{1}\|Y\|_{L_{p e r}^{2}}\left(\left\|\Psi_{c}\right\|_{H_{p e r}^{1}}+\epsilon\right)}{\left(\mathcal{H}\left(\Psi_{c}\right)-\mathcal{H}\left(U_{0}\right)\right)}<L .
$$

In other words, the solution leaves the tube before reaching the period $L$, and so the $\Psi_{c}$-orbit is unstable.

3. Existence of smooth curves of periodic traveling waves $(n=2)$

In this section we are interested in building explicit traveling wave solutions $\Psi_{c}=$ $\left(\psi_{c}, p_{c}\right)$ for the system (1.1) for $n=2$. Our analysis will show that the initial profile of $\psi_{c}$ could be of either snoidal type for $c \in(1, \sqrt{a / b})$, or cnoidal type for $c \in(\sqrt{a / b}, 1)$. Our main interest here will be the construction of smooth curves $c \rightarrow \psi_{c}$ of periodic traveling waves with a fixed fundamental period $L$, depending on the range of the wave speed $c$. 
3.1. Building periodic solutions. One can see directly that if we look for traveling wave solutions for the system (1.1), i.e. solutions of the form

$$
(q(x, t), p(x, t))=\left(q_{c}(x-c t), p_{c}(x-c t)\right),
$$

with $r_{c}=-c q_{c}$, then $q_{c}$ has to satisfy the equation

$$
\left(a-b c^{2}\right) \psi^{\prime \prime}+\left(c^{2}-1\right) \psi-\frac{4 c}{3} \psi^{3}=A_{0}
$$

For the propose of this paper, we will consider solutions for which we have that $A_{0}=0$. In other words, we look for functions $q_{c}$ satisfying the equation

$$
\left(a-b c^{2}\right) \psi^{\prime \prime}+\left(c^{2}-1\right) \psi-\frac{4 c}{3} \psi^{3}=0
$$

Case I. $c \in I_{1}=(1, \sqrt{a / b})$.

We start by scaling $\psi$ on this range of the wave speed. Define

$$
\psi_{1}(x)=\beta_{1}(c) \varphi_{1}\left(\theta_{1}(c) x\right), \text { with } \beta_{1}(c)=\frac{3}{2 c} \sqrt{c^{2}-1} \text { and } \theta_{1}^{2}(c)=\frac{3\left(c^{2}-1\right)}{c\left(a-b c^{2}\right)} .
$$

Then $\varphi_{1}$ satisfies the ordinary differential equation

$$
\Lambda_{1, c}\left(\varphi_{1}\right)=\varphi_{1}^{\prime \prime}-\varphi_{1}^{3}+\frac{c}{3} \varphi_{1}=0 .
$$

Multiplying by $\varphi_{1}^{\prime}$ and integrating over $[0, x]$ we see that $\varphi_{1}$ already satisfies the equation

$$
\frac{1}{2}\left(\varphi_{1}^{\prime}\right)^{2}-\frac{1}{4} \varphi_{1}^{4}+\frac{c}{6} \varphi_{1}^{2}=D_{1}
$$

with $D_{1}=D\left(\varphi_{1}\right)$ being an integration constant. In other words, $\varphi^{\prime}$ has to satisfy the quadrature form

$$
\left(\varphi_{1}^{\prime}\right)^{2}=\frac{1}{2}\left(\varphi_{1}^{4}-\frac{2 c}{3} \varphi_{1}^{2}+4 D_{1}\right)
$$

Note that formula (3.4) may be written as

$$
\left[\varphi_{1}^{\prime}(z)\right]^{2}=\frac{1}{2} P_{1}\left(\varphi_{1}(z)\right),
$$

where $P_{1}$ is the fourth degree polynomial $P_{1}(t)=t^{4}-\frac{2 c}{3} t^{2}+4 D_{1}$. By supposing that $P_{1}$ has only real root, say $-\eta_{2}<-\eta_{1}<0<\eta_{1}<\eta_{2}$, one sees that

$$
P_{1}(t)=\left(\eta_{1}^{2}-t^{2}\right)\left(\eta_{2}^{2}-t^{2}\right)
$$

and that (3.5) can be written

$$
\left(\varphi_{1}^{\prime}\right)^{2}=\frac{1}{2}\left(\eta_{1}^{2}-\varphi_{1}^{2}\right)\left(\eta_{2}^{2}-\varphi_{1}^{2}\right)
$$

with $\eta_{1}$ and $\eta_{2}$ satisfying conditions

$$
\left\{\begin{array}{l}
\eta_{1}^{2}+\eta_{2}^{2}=\frac{2 c}{3} \\
\eta_{1}^{2} \eta_{2}^{2}=4 D_{1}
\end{array}\right.
$$


It follows immediately from (3.5)-(3.6) that $\varphi_{1}$ must take values in the range $-\eta_{2}<$ $-\eta_{1} \leq \varphi_{1} \leq \eta_{1}<\eta_{2}$. Change variables again by letting

$$
\varphi_{1}(x)=\eta_{1} \varrho(\zeta), \quad \zeta=\frac{\eta_{2}}{\sqrt{2}} x
$$

with $\varrho(0)=0$ and $\varrho$ continuous. Then substituting into (3.7) yields the equation

$$
\left(\varrho^{\prime}\right)^{2}=\left(1-\varrho^{2}\right)\left(1-k_{1}^{2} \varrho^{2}\right),
$$

where the modulus $0<k_{1}<1$ is defined as

$$
k_{1}^{2}=\frac{\eta_{1}^{2}}{\eta_{2}^{2}}
$$

But from formula 128.01 of [7], we have that the solution is $\varrho\left(\zeta, \eta_{1}, \eta_{2}\right)=\operatorname{sn}\left(\zeta ; k_{1}\right)$. As a consequence of this we have that equation (3.3) has a snoidal wave solution of the form

$$
\varphi_{1}\left(x, \eta_{1}, \eta_{2}\right)=\eta_{1} \operatorname{sn}\left(\frac{\eta_{2}}{\sqrt{2}} x ; k_{1}\right) .
$$

It is well known that sn is a $4 K$ periodic function where $K$ is the elliptic integral of the first class defined by

$$
K\left(k_{1}\right)=\int_{0}^{\pi / 2} \frac{d t}{\sqrt{1-k_{1}^{2} \sin ^{2} t}} .
$$

Thus $\varphi_{1}$ has fundamental period

$$
T_{\varphi_{1}}=\frac{4 \sqrt{2}}{\eta_{2}} K\left(k_{1}\right) .
$$

Recall that for given $c>0$, we have that $0<\eta_{1}<\eta_{2}<\sqrt{2 c / 3}$, so that $\eta_{2}=\sqrt{2 \lambda-\eta_{1}^{2}}$ where $\lambda=c / 3$. Then we see that the period $T_{\varphi_{1}}:=T_{1}$ and the modulus $k_{1}$ depend on $\eta_{1}:=\eta$

$$
T_{1}(\eta)=\frac{4 \sqrt{2}}{\sqrt{2 \lambda-\eta^{2}}} K\left(k_{1}(\eta)\right), \quad k_{1}^{2}(\eta)=\frac{\eta^{2}}{2 \lambda-\eta^{2}}
$$

In particular, if $\eta \rightarrow 0$, then $k_{1} \rightarrow 0$. This implies that $K\left(k_{1}\right) \rightarrow \pi / 2$, and so $T_{1} \rightarrow \frac{2}{\sqrt{\lambda}} \pi$.

On the other hand, if $\eta \rightarrow \sqrt{\lambda}$, then $k_{1} \rightarrow 1$ and $K\left(k_{1}\right) \rightarrow \infty$ implying that $T_{1} \rightarrow \infty$. But the function $\eta \rightarrow T_{1}(\eta)$ is an increasing function for $0<\eta<\sqrt{\lambda}$. In fact,

$$
\frac{d T_{1}}{d \eta}=4 \sqrt{2}\left(\left(2 \lambda-\eta^{2}\right)^{-3 / 2} \eta K\left(k_{1}(\eta)\right)+\left(2 \lambda-\eta^{2}\right)^{-1 / 2} \frac{d K}{d k_{1}} \frac{d k_{1}}{d \eta}\right) .
$$

Using that

$$
\frac{d k_{1}}{d \eta}=\frac{2 \lambda \eta}{k_{1}\left(2 \lambda-\eta^{2}\right)^{2}}
$$


we obtain

$$
\frac{d T_{1}}{d \eta}=\frac{4 \sqrt{2} \eta}{\left(2 \lambda-\eta^{2}\right)^{5 / 2}}\left(\left(2 \lambda-\eta^{2}\right) K\left(k_{1}(\eta)\right)+\frac{2 \lambda}{k_{1}} \frac{d K}{d k_{1}}\right) .
$$

Since $K$ is a strictly increasing function on $k_{1}$ and $2 \lambda-\eta^{2}>0$, we conclude that $T_{1}$ is an increasing function for $0<\eta<\sqrt{\lambda}$. In particular, we have that

$$
T_{1}(\eta)>\frac{2 \pi}{\sqrt{\lambda}}
$$

In terms of $\eta$ and $\lambda$, the solution takes the form

$$
\psi_{1}\left(x, \eta_{1}, \eta_{2}\right)=\frac{3 \eta \sqrt{c^{2}-1}}{2 c} \operatorname{sn}\left(\sqrt{\frac{3\left(2 \lambda-\eta^{2}\right)\left(c^{2}-1\right)}{2 c\left(a-b c^{2}\right)}} x ; k_{1}\right)
$$

We also can consider the degenerates cases $\eta \rightarrow \sqrt{\lambda}$ and $\eta \rightarrow 0$. In the first case, we have that the period tends to infinity and $k_{1} \rightarrow 1$. Thus

$$
\psi_{1}(x) \rightarrow \frac{1}{2} \sqrt{\frac{3\left(c^{2}-1\right)}{c}} \operatorname{sn}\left(\sqrt{\frac{\left(c^{2}-1\right)}{2\left(a-b c^{2}\right)}} x ; 1\right)=\frac{1}{2} \sqrt{\frac{3\left(c^{2}-1\right)}{c}} \tanh \left(\sqrt{\frac{\left(c^{2}-1\right)}{2\left(a-b c^{2}\right)}} x\right),
$$

which is a traveling wave solution for equation (3.1) in the real line. In the second case, $\psi_{1} \rightarrow 0$, which is one of the constant traveling wave solutions.

Case II. $c \in I_{2}=(\sqrt{a / b}, 1)$.

To scale $\psi$ on this range of the wave speed, we define

$$
\psi_{2}(x)=\beta_{2}(c) \varphi_{2}\left(\theta_{2}(c) x\right) \text {, with } \beta_{2}(c)=\frac{3}{2 c} \sqrt{1-c^{2}} \text { and } \theta_{2}^{2}(c)=\frac{3\left(1-c^{2}\right)}{c\left(b c^{2}-a\right)} .
$$

Then $\varphi_{2}$ satisfies the ordinary differential equation

$$
\Lambda_{2, c}\left(\varphi_{2}\right)=\varphi_{2}^{\prime \prime}+\varphi_{2}^{3}+\frac{c}{3} \varphi_{2}=0
$$

As in Case I, we have that equation (3.12) has a cnoidal wave solution of the form

$$
\varphi_{2}\left(x, \eta_{1}, \eta_{2}\right)=\eta_{1} \mathrm{cn}\left(\sqrt{\frac{\eta_{1}^{2}+\eta_{2}^{2}}{2}} x ; k_{2}\right)
$$

where $\eta_{1}$ and $\eta_{2}$ satisfy conditions

$$
\left\{\begin{array}{l}
\eta_{2}^{2}-\eta_{1}^{2}=\frac{2 c}{3} \\
\eta_{1}^{2} \eta_{2}^{2}=4 D_{2}
\end{array}\right.
$$

and the modulus $0<k_{2}<\sqrt{1 / 2}$ is defined as

$$
k_{2}^{2}=\frac{\eta_{1}^{2}}{\eta_{1}^{2}+\eta_{2}^{2}}, \quad k_{2}^{\prime 2}=1-k_{2}^{2}
$$

Now, since cn is a $4 K$ periodic function, $\varphi_{2}$ has fundamental period

$$
T_{\varphi_{2}}=\frac{4 \sqrt{2}}{\sqrt{\eta_{1}^{2}+\eta_{2}^{2}}} K\left(k_{2}\right)
$$


Now from the definition $\eta_{2}=\sqrt{2 \lambda+\eta_{1}^{2}}$ where $\lambda=c / 3$ and the period $T_{\varphi_{2}}:=T_{2}$ and the modulus $k_{2}$ depend only on $\eta=\eta_{1}$,

$$
T_{2}(\eta)=\frac{4}{\sqrt{\lambda+\eta^{2}}} K\left(k_{2}(\eta)\right), \quad k_{2}^{2}(\eta)=\frac{\eta^{2}}{2\left(\lambda+\eta^{2}\right)} .
$$

It is not hard to see that $T_{\varphi_{2}}$ is a decreasing function for $\eta>0$. So we conclude that

$$
0<T_{2}(\eta)<\frac{2 \pi}{\sqrt{\lambda}}
$$

In terms of $\eta$ and $\lambda$, the solution takes the form

$$
\psi_{2}(x, \eta)=\frac{3 \eta \sqrt{1-c^{2}}}{2 c} \operatorname{cn}\left(\sqrt{\eta^{2}+\lambda} \sqrt{\frac{3\left(1-c^{2}\right)}{c\left(b c^{2}-a\right)}} x ; k_{2}\right) .
$$

If we consider the degenerate case $\eta \rightarrow 0$, then $k_{2} \rightarrow 0$ and $K \rightarrow \pi / 2$. In this case we capture the unique constant solution $\psi_{2} \equiv 0$.

3.2. Characterization of the fundamental period. A clever result needed in discussing orbital instability is the existence of smooth curves of periodic traveling wave solutions with a fixed period, which are parametrized by the wave speed $c$. We want to point out that this is not straightforward since the period of these periodic traveling waves solutions depend strongly on the wave speed $c$. We will see that the proof of these facts is a direct consequence of the Implicit Function Theorem. We start the discussion by characterizing the period.

Lemma 3.1. Let $L>2 \sqrt{3} \pi$ be arbitrary but fixed. Consider $1<c_{0}^{2}<a / b$ such that $c_{0}>\frac{12 \pi^{2}}{L^{2}}$, and a unique $\eta_{1,0}=\eta_{1}\left(c_{0}\right) \in\left(0, \sqrt{\frac{c_{0}}{3}}\right)$ such that $L=T_{c_{0}, 1}$. Let $\varphi_{c_{0}}(\cdot)=$ $\varphi_{c_{0}}\left(\cdot, \eta_{1,0}, \eta_{2,0}\right)$ satisfy the equation (3.3) with $\eta_{2,0}=\sqrt{\frac{2 c_{0}}{3}-\eta_{1,0}^{2}}$, and define

$$
\rho(c)=\sqrt{\frac{\left(a-b c_{0}^{2}\right)\left(c^{2}-1\right)}{\left(a-b c^{2}\right)\left(c_{0}^{2}-1\right)}}
$$

for $c>0$ such that $1<c^{2}<a / b$. Then

1. There is an interval $J_{1}\left(c_{0}\right)$ around $c_{0}$, an interval $B\left(\eta_{2,0}\right)$ around $\eta_{1,0}$, and a unique smooth function $\eta: J_{1}\left(c_{0}\right) \longrightarrow B\left(\eta_{1,0}\right)$ satisfying that $\eta\left(c_{0}\right)=\eta_{1,0}$ and

$$
\sqrt{\frac{c}{c_{0}}} \frac{4 \sqrt{2}}{\sqrt{2 c / 3-\eta^{2}}} K\left(k_{1}(\eta)\right)=\rho(c) T_{c_{0}, 1}
$$

where $k_{1}^{2}(c)=\frac{\eta^{2}}{2 c / 3-\eta^{2}}$.

2. The snoidal wave $\varphi_{c}(\cdot)=\varphi_{c_{0}}\left(\cdot, \sqrt{\frac{c_{0}}{c}} \eta_{1}(c), \sqrt{\frac{c_{0}}{c}} \eta_{2}(c)\right)$, where $\eta=\eta_{1}$ and $\eta_{2}=$ $\sqrt{\frac{2 c}{3}-\eta^{2}}$, has fundamental period $T_{c}=\rho(c) T_{c_{0}, 1}$ and satisfies the equation

$$
\varphi^{\prime \prime}+\frac{c_{0}}{3} \varphi-\varphi^{3}=0
$$


Lemma 3.2. Let $L<2 \sqrt{3} \pi$ be arbitrary but fixed. Consider $a / b<c_{0}^{2}<1$ such that $c_{0}<\frac{12 \pi^{2}}{L^{2}}$, and a unique $\eta_{1,0}=\eta_{1}\left(c_{0}\right) \in\left(0, \sqrt{\frac{2 c_{0}}{3}}\right)$ such that $L=T_{c_{0}, 2}$. Let $\varphi_{c_{0}}(\cdot)=$ $\varphi_{c_{0}}\left(\cdot, \eta_{1,0}, \eta_{2,0}\right)$ satisfy the equation (3.12) with $\eta_{2,0}=\sqrt{\frac{2 c_{0}}{3}+\eta_{1,0}^{2}}$, and define

$$
\rho(c)=\sqrt{\frac{\left(b c_{0}^{2}-a\right)\left(1-c^{2}\right)}{\left(b c^{2}-a\right)\left(1-c_{0}^{2}\right)}}
$$

for $c>0$ such that $a / b<c^{2}<1$. Then

1. There is an interval $J_{2}\left(c_{0}\right)$ around $c_{0}$, an interval $B\left(\eta_{2,0}\right)$ around $\eta_{2,0}$, and a unique smooth function $\eta: J_{2}\left(c_{0}\right) \longrightarrow B\left(\eta_{2,0}\right)$ satisfying that $\eta\left(c_{0}\right)=\eta_{1,0}$ and

$$
\sqrt{\frac{c}{c_{0}}} \frac{4}{\sqrt{c / 3+\eta^{2}}} K\left(k_{2}\right)=\rho(c) T_{c_{0}, 2},
$$

where $k_{2}^{2}(c)=\frac{\eta^{2}}{2\left(c / 3+\eta^{2}\right)}$.

2. The cnoidal wave $\varphi_{c}(\cdot)=\varphi_{c_{0}}\left(\cdot, \sqrt{\frac{c_{0}}{c}} \eta_{1}(c), \sqrt{\frac{c_{0}}{c}} \eta_{2}(c)\right)$, where $\eta=\eta_{1}$ and $\eta_{2}=$ $\sqrt{\frac{2 c}{3}+\eta^{2}}$, has fundamental period $T_{c}=\rho(c) T_{c_{0}, 2}$ and satisfies the equation

$$
\varphi^{\prime \prime}+\frac{c_{0}}{3} \varphi+\varphi^{3}=0
$$

Proof of these lemmas follows by noting that we can define, in an appropriate range for $c$, functions

$$
\begin{aligned}
& \Pi_{1}(\eta, c)=\sqrt{\frac{c}{c_{0}}} \frac{4 \sqrt{2}}{\sqrt{2 \lambda-\eta^{2}}} K\left(k_{1}(\eta)\right)-\rho(c) T_{c_{0}, 1} \text { in } \Omega_{1}=\left\{(\eta, c): 0<\eta<\sqrt{\frac{c}{3}}, c>\frac{12 \pi^{2}}{T_{c_{0}, 1}^{2}}\right\}, \\
& \Pi_{2}(\eta, c)=\sqrt{\frac{c}{c_{0}}} \frac{4}{\sqrt{\lambda+\eta^{2}}} K\left(k_{2}(\eta)\right)-\rho(c) T_{c_{0}, 2} \text { in } \Omega_{2}=\left\{(\eta, c): 0<\eta<\sqrt{\frac{2 c}{3}}, c<\frac{12 \pi^{2}}{T_{c_{0}, 2}^{2}}\right\},
\end{aligned}
$$

where $\lambda=c / 3$. First observe that $\Pi_{i}(\eta, c)=\sqrt{\frac{c}{c_{0}}} T_{i}(\eta)-\rho(c) T_{c_{0}, i}$ (see (3.16), (3.18)). We also have that $\Pi_{i}\left(\eta_{0}, c_{0}\right)=0$ and that $\partial_{\eta} \Pi_{i}\left(\eta_{0}, c_{0}\right)=\sqrt{\frac{c}{c_{0}}} \partial_{\eta} T_{i}\left(\eta_{0}\right) \neq 0$, since we show in these three cases that the period function $T_{i}$ is a monotone function for $0<\eta<$ $\sqrt{\frac{c}{3}}$ for $i=1$ and for $0<\eta<\sqrt{\frac{2 c}{3}}$ for $i=2$. So, the first part in these lemmas follows by the Implicit Function Theorem. The second part in these lemmas is straightforward.

3.3. Curves of periodic solutions. As a consequence of the previous results, we will prove the existence of smooth curves of periodic waves for equation (3.1) with a fixed period characterized as $T_{0, i}=\frac{T_{c_{0, i}}}{\theta_{i}\left(c_{0}\right)}$ for $c \in J_{i}\left(c_{0}\right) \bigcap I_{i}$ with $i=1,2$.

These curves of periodic wave solutions are given by the following result

THEOREM 3.3. For any $c \in J_{i}\left(c_{0}\right)$, there exists $\psi_{c, i} \in \mathcal{W}_{L}^{1}$ with fundamental period $L=T_{0, i}$ satisfying the equation

$$
\left(a-b c^{2}\right) \psi^{\prime \prime}+\left(c^{2}-1\right) \psi-\frac{4 c}{3} \psi^{3}=0
$$


and having the form

$$
\begin{aligned}
& \psi_{c, 1}(x)=\frac{3 \eta_{1} \sqrt{c^{2}-1}}{2 c} \operatorname{sn}\left(\eta_{2} \sqrt{\frac{3\left(c^{2}-1\right)}{2 c\left(a-b c^{2}\right)}} x ; k_{1}\right), \quad \text { with } k_{1}^{2}=\frac{\eta_{1}^{2}}{\eta_{2}^{2}}, \quad \eta_{1}^{2}+\eta_{2}^{2}=\frac{2 c}{3}, \\
& \psi_{c, 2}(x)=\frac{3 \eta_{1} \sqrt{1-c^{2}}}{2 c} \operatorname{cn}\left(\sqrt{\eta_{1}^{2}+\eta_{2}^{2}} \sqrt{\frac{3\left(1-c^{2}\right)}{2 c\left(b c^{2}-a\right)}} x ; k_{2}\right), \text { with } k_{2}^{2}=\frac{\eta_{1}^{2}}{\eta_{1}^{2}+\eta_{2}^{2}}, \eta_{2}^{2}-\eta_{1}^{2}=\frac{2 c}{3} .
\end{aligned}
$$

for $i=1,2$, respectively.

Moreover, the map

$$
\begin{aligned}
J_{i}\left(c_{0}\right) & \rightarrow \mathcal{W}_{L}^{1} \times H_{p e r}^{1}[0, L], \\
c & \rightarrow\left(\psi_{c, i},-c B\left(\psi_{c, i}\right)+\frac{1}{3} \psi_{c, i}^{3}\right)
\end{aligned}
$$

is smooth $(i=1,2)$.

Proof. Let $\varphi_{c, i}$ be the periodic wave determined by the corresponding previous lemmas with $\eta_{i}=\eta_{i}(c)$ for $c \in J_{i}\left(c_{0}\right)$. Define

$$
\varphi_{i}(x)=\sqrt{\frac{c}{c_{0}}} \varphi_{c, i}\left(\sqrt{\frac{c}{c_{0}}} x\right), \quad x \in \mathbb{R} .
$$

Then $\varphi_{i}$ has period $T_{\varphi_{i}} \equiv \gamma(c) T_{c_{0}, i}$ with

$$
\gamma(c)=\frac{\theta_{i}(c)}{\theta_{i}\left(c_{0}\right)} .
$$

But a direct computation shows that $\varphi_{i}$ satisfies the equation

$$
\Lambda_{i, c}\left(\varphi_{i}\right)=0 .
$$

Next we obtain a smooth curve of the solution for (3.1). From the change of variables in the $i$-case, we define

$$
\psi_{c, i}(x)=\beta_{i}(c) \varphi_{i}\left(\theta_{i}(c) x, \eta_{1}(c), \eta_{2}(c)\right) .
$$

Then it is a straightforward calculation to see that $\psi_{c, i}$ has period $T_{0, i}=\frac{T_{c_{0, i}}}{\theta_{i}\left(c_{0}\right)}$ and that $\psi_{c, i}$ has the form desired in the conclusion of the Theorem for $i=1,2$. Regularity of the map $c \rightarrow \psi_{c, i}$ follows from the properties of $\varphi_{c_{0}, i}$ and $\eta_{j}$.

REMARK 3.4. By a continuation argument, $J_{i}$ in the previous result can be taken as

$$
J_{1}=(1, \sqrt{a / b}), \quad J_{2}=(\sqrt{a / b}, 1) .
$$

\section{Instability result for $n=2$}

We start proving a basic result before we follow the approach in [9]. First note that $\mathcal{L}_{c, n}=\mathcal{F}^{\prime \prime}\left(\Psi_{c}\right)$ is given by

$$
\mathcal{L}_{c, n}=\left(\begin{array}{cc}
n c q_{c}^{n}+B^{-1}\left(q_{c}^{n}(\cdot)\right) q_{c}^{n}+A & -q_{c}^{n} B^{-1}+c I \\
-B^{-1}\left(q_{c}^{n}(\cdot)\right)+c I & B^{-1}
\end{array}\right) .
$$


So, it is straightforward to see that

$$
\begin{array}{r}
\left\langle\mathcal{L}_{c, n}\left(\Psi_{c}\right), \Psi_{c}\right\rangle=\int_{0}^{L}\left[n c q_{c}^{n+2}+q_{c} A q_{c}+q_{c}^{n+1} B^{-1}\left(q_{c}^{n+1}\right)-2 q_{c}^{n+1} B^{-1}\left(p_{c}\right)\right. \\
\left.+p_{c} B^{-1}\left(p_{c}\right)+2 c p_{c} q_{c}\right] d x .
\end{array}
$$

Now on the other hand, a direct computation shows that

$$
\begin{aligned}
q_{c}^{n+1} B^{-1}\left(p_{c}\right) & =\frac{1}{n+1} q_{c}^{n+1} B^{-1}\left(q_{c}^{n+1}\right)-c q_{c}^{n+2} \\
2 c p_{c} q_{c} & =\frac{2 c}{n+1} q_{c}^{n+2}-2 c^{2} q_{c} B q_{c} \\
p_{c} B^{-1}\left(p_{c}\right)= & \frac{1}{(n+1)^{2}} q_{c}^{n+1} B^{-1}\left(q_{c}^{n+1}\right)-\frac{2 c}{n+1} q_{c}^{n+2}+c^{2} q_{c} B q_{c} \\
\frac{1}{(n+1)^{2}} q_{c}^{n+1} B^{-1}\left(q_{c}^{n+1}\right)= & \frac{1}{(n+2)^{2} c^{2}}\left[c^{4} q_{c} B q_{c}-2 c^{2} q_{c} A q_{c}+A q_{c} B^{-1}\left(A q_{c}\right)\right. \\
& \left.+\left(-c^{2} B q_{c}+A q_{c}+A_{1}-c^{2} q_{c}+B^{-1}\left(A q_{c}\right)\right) A_{1}\right] .
\end{aligned}
$$

Using that $q_{c}$ has zero mean in $[0, L]$ we have that

$$
\begin{aligned}
& \frac{n^{2}}{(n+1)^{2}} \int_{0}^{L} q_{c}^{n+1} B^{-1}\left(q_{c}^{n+1}\right) d x \\
= & \frac{n^{2} A_{1}^{2} L}{(n+2)^{2} c^{2}}+\frac{n^{2}}{(n+2)^{2} c^{2}} \int_{0}^{L}\left[c^{4} q_{c} B q_{c}-2 c^{2} q_{c} A q_{c}+A q_{c} B^{-1}\left(A q_{c}\right)\right] d x .
\end{aligned}
$$

As a consequence of these equalities, we have that

$$
\begin{aligned}
\left\langle\mathcal{L}_{c, n}\left(\Psi_{c}\right), \Psi_{c}\right\rangle & =\int_{0}^{L}\left[(n+2) c q_{c}^{n+2}+q_{c} A q_{c}-c^{2} q_{c} B q_{c}+\frac{n^{2}}{(n+1)^{2}} q_{c}^{n+1} B^{-1}\left(q_{c}^{n+1}\right)\right] d x \\
& =\frac{n^{2} A_{1}^{2} L}{(n+2)^{2} c^{2}}+\int_{0}^{L}\left[n\left(c^{2} q_{c} B q_{c}-q_{c} A q_{c}\right)+\frac{n^{2}}{(n+2)^{2} c^{2}}\left[c^{4} q_{c} B q_{c}\right.\right. \\
& \left.\left.\quad-2 c^{2} q_{c} A q_{c}+A q_{c} B^{-1}\left(A q_{c}\right)\right]\right] d x \\
& =\frac{n^{2} A_{1}^{2} L}{(n+2)^{2} c^{2}}+\frac{n}{(n+2)^{2} c^{2}} \int_{0}^{L}\left[\left[n+(n+2)^{2}\right] c^{4} q_{c} B q_{c}\right. \\
& =\frac{n^{2} A_{1}^{2} L}{(n+2)^{2} c^{2}}+\frac{n}{(n+2)^{2} c^{2}} I_{1}
\end{aligned}
$$

where

$$
I_{1}=\int_{0}^{L}\left[\left[n+(n+2)^{2}\right] c^{4} q_{c} B q_{-}\left[2 n+(n+2)^{2}\right] c^{2} q_{c} A q_{c}+n A q_{c} B^{-1}\left(A q_{c}\right)\right] d x .
$$

Moreover, for $n$ even we can take $A_{1}=0$ (see case $n=2$ in Section 3), and so

$$
\left\langle\mathcal{L}_{c, n}\left(\Psi_{c}\right), \Psi_{c}\right\rangle=\frac{n}{(n+2)^{2} c^{2}} I_{1} .
$$


Now we observe for $\hat{\rho}_{k}=\frac{4 \pi^{2} \rho k^{2}}{L^{2}}$, with $\rho=a, b$ that

$$
\begin{aligned}
& \frac{I_{1}}{L}= \frac{1}{L} \int_{0}^{L}\left[\left[n+(n+2)^{2}\right] c^{4} q_{c} B q_{c}-\left[2 n+(n+2)^{2}\right] c^{2} q_{c} A q_{c}+n A q_{c} B^{-1}\left(A q_{c}\right)\right] d x \\
&=\sum_{k \neq 0}\left[\left[n+(n+2)^{2}\right] c^{4}\left[1+\frac{4 \pi^{2} b k^{2}}{L^{2}}\right]-\left[2 n+(n+2)^{2}\right] c^{2}\left[1+\frac{4 \pi^{2} a k^{2}}{L^{2}}\right]\right. \\
&\left.\quad+n \frac{\left.\left[1+\frac{4 \pi^{2} a k^{2}}{L^{2}}\right]^{2}\right]}{\left[1+\frac{4 \pi^{2} b k^{2}}{L^{2}}\right]}\right]\left|\hat{q}_{k}\right|^{2} \\
& \quad \\
& \quad \sum_{k \neq 0} \frac{\left[\left[n+(n+2)^{2}\right]\left(1+\hat{b}_{k}\right)^{2} c^{4}-\left[2 n+(n+2)^{2}\right]\left(1+\hat{b}_{k}\right)\left(1+\hat{a}_{k}\right) c^{2}+n\left(1+\hat{a}_{k}\right)^{2}\right]}{\left(1+\hat{b}_{k}\right)}\left|\hat{q}_{k}\right|^{2} .
\end{aligned}
$$

Now, a simple computation shows that

$$
\begin{aligned}
& \left(n+(n+2)^{2}\right)\left(1+\hat{b}_{k}\right)^{2} c^{4}-\left(2 n+(n+2)^{2}\right)\left(1+\hat{b}_{k}\right)\left(1+\hat{a}_{k}\right) c^{2}+n\left(1+\hat{a}_{k}\right)^{2} \\
= & \left(n+(n+2)^{2}\right)\left(1+\hat{b}_{k}\right)^{2}\left(c^{2}-\frac{\left(1+\hat{a}_{k}\right)}{\left(1+\hat{b}_{k}\right)}\right)\left(c^{2}-\frac{n\left(1+\hat{a}_{k}\right)}{\left(n+(n+2)^{2}\right)\left(1+\hat{b}_{k}\right)}\right) .
\end{aligned}
$$

If we assume for example that $1>\frac{b}{a}>\frac{n}{n+(n+2)^{2}}$ and that $1<|c|<\sqrt{\frac{1+\frac{4 \pi^{2} a}{L^{2}}}{1+\frac{4 \pi^{2} b}{L^{2}}}}$, then we have for any $k \in \mathbb{Z} \backslash\{0\}$ that

$$
\left(c^{2}-\frac{\left(1+\hat{a}_{k}\right)}{\left(1+\hat{b}_{k}\right)}\right)\left(c^{2}-\frac{n\left(1+\hat{a}_{k}\right)}{\left(n+(n+2)^{2}\right)\left(1+\hat{b}_{k}\right)}\right)<0 .
$$

This follows since for $a>b$ and $k \in \mathbb{Z} \backslash\{0\}$, we have that

$$
\sqrt{\frac{1+\frac{4 \pi^{2} a}{L^{2}}}{1+\frac{4 \pi^{2} b}{L^{2}}}} \leq \sqrt{\frac{1+\hat{a}_{k}}{1+\hat{b}_{k}}}<\sqrt{\frac{a}{b}} .
$$

On the other hand,

$$
|c|>1=\sqrt{\frac{a}{b}} \sqrt{\frac{b}{a}}>\sqrt{\frac{n\left(1+\hat{a}_{k}\right)}{\left(n+(n+2)^{2}\right)\left(1+\hat{b}_{k}\right)}},
$$

which implies that

$$
\sqrt{\frac{n\left(1+\hat{a}_{k}\right)}{\left(n+(n+2)^{2}\right)\left(1+\hat{b}_{k}\right)}}<|c|<\sqrt{\frac{1+\hat{a}_{k}}{1+\hat{b}_{k}}} .
$$

In other words, for $n$ even we have established the instability hypothesis (H3).

LEMmA 4.1. Let $n$ be even. If $1>\frac{b}{a}>\frac{n}{\left(n+(n+2)^{2}\right)}$ and $1<c^{2}<\frac{1+\frac{4 \pi^{2} a}{L^{2}}}{1+\frac{4 \pi^{2} b}{L^{2}}}$, then we have that

$$
\left\langle\mathcal{L}_{c, n}\left(\Psi_{c}\right), \Psi_{c}\right\rangle<0
$$


4.1. Spectral Analysis of $\mathcal{L}_{c, n}$ for $1<c<\sqrt{a / b}$ and $n=2$. We will see below that the spectral analysis is based on the fact that $\mathcal{L}_{c, n}$ can be diagonalized. In fact, set $D$ as the linear transformation

$$
D=\left(\begin{array}{cc}
I & 0 \\
q_{c}^{n}-c B & I
\end{array}\right)
$$

whose transpose operator $D^{t}$ is given by

$$
D^{t}=\left(\begin{array}{lc}
I & q_{c}^{n}-c B \\
0 & I
\end{array}\right)
$$

It is not hard to show that $D$ is a bounded linear operator from $H_{p e r}^{s}([0, L]) \times$ $H_{\text {per }}^{s-2}([0, L]) \rightarrow H_{\text {per }}^{s}([0, L]) \times H_{\text {per }}^{s-2}([0, L])$, with bounded inverse. On the other hand,

$$
\mathcal{L}_{c}^{(n)}=D^{t} \mathcal{F}^{\prime \prime}\left(\begin{array}{l}
q_{c} \\
p_{c}
\end{array}\right) D=\left(\begin{array}{cc}
\mathcal{L}_{s n} & 0 \\
0 & B^{-1}
\end{array}\right)
$$

where $\mathcal{L}_{s n}=A-c^{2} B+(n+2) c q_{c}^{n}$. From this diagonal form the spectral analysis of the operator $\mathcal{L}_{c, 2}$, is reduced to the analysis of the spectral properties of the operator

$$
\mathcal{L}_{c}^{(2)}=\left(\begin{array}{cc}
\mathcal{L}_{s n} & 0 \\
0 & B^{-1}
\end{array}\right)
$$

where

$$
\mathcal{L}_{s n}=-\left(a-b c^{2}\right) \frac{d^{2}}{d x^{2}}+\left(1-c^{2}+4 c q_{c, 1}^{2}\right)
$$

is the operator associated to the snoidal-wave solution $\left(q_{c, 1}, p_{c, 1}\right)^{t}$ for system (1.1) determined by Theorem (3.3). As a consequence of this result, now we are able to complete the spectral analysis for $\mathcal{L}_{c, 2}$. In other words, we have to consider the periodic eigenvalue problem

$$
\left\{\begin{array}{l}
\mathcal{L}_{c, 2} \chi=\lambda \chi \\
\chi(0)=\chi(L), \chi^{\prime}(0)=\chi^{\prime}(L)
\end{array}\right.
$$

where the operator $\mathcal{L}_{c, 2}$ is given by

$$
\mathcal{L}_{c, 2}=\left(\begin{array}{cc}
2 q_{c, 1}^{2}+B^{-1}\left(q_{c, 1}^{2}(\cdot)\right) q_{c, 1}^{2}+A & -q_{c, 1}^{2} B^{-1}+c I \\
-B^{-1}\left(q_{c, 1}^{2}(\cdot)\right)+c I & B^{-1}
\end{array}\right) .
$$

Regarding this eigenvalue problem, we obtain the following result

TheOREM 4.2. Let $q_{c, 1}$ be the snoidal wave solution given by Theorem 2.4. and $1<c<\sqrt{a / b}$. Then the periodic eigenvalue problem (4.3) on $H_{\text {per }}^{2}([0, L]) \times H_{\text {per }}^{2}([0, L])$ has exactly one negative eigenvalue which is simple, $\lambda=0$ is a simple eigenvalue with eigenfunction $\left(q_{c, 1}^{\prime}, 0\right)^{t}$ and the rest of the spectrum is positive and away from zero.

Proof. Since $B^{-1}$ is a positive operator, we only need to analyze the periodic eigenvalue problem for the linear operator $\mathcal{L}_{s n}$ :

$$
\left\{\begin{array}{l}
\mathcal{L}_{s n} v=\lambda v \\
v(0)=v(L), \quad v^{\prime}(0)=v^{\prime}(L) .
\end{array}\right.
$$


Recall that $\mathcal{L}_{s n} v=\lambda v$ is simply a Hill's equation, so it is not hard to check (see [12]) that the linear operator $\mathcal{L}_{s n}$ in $(4.4)$ defined on $H_{\text {per }}^{2}([0, L])$ has exactly its first three eigenvalues simple: the first eigenvalue is negative, the second eigenvalue is zero with eigenfunction $q_{c, 1}^{\prime}$, and the third eigenvalue is positive. The rest of the spectrum is a discrete set of eigenvalues which are double. The eigenvalues only accumulate at $+\infty$. Now, let $\zeta=\left(q_{c, 1}^{\prime}, 0\right)^{t}$. Then we have that $\mathcal{L}_{c}^{(2)} \zeta=\left(\mathcal{L}_{s n} q_{c}^{\prime}, 0\right)^{t}=(0,0)^{t}$. In other words, $\lambda=0$ is an eigenvalue of $\mathcal{L}_{c}^{(2)}$ with eigenvector $\zeta=\left(\psi_{c}^{\prime}, 0\right)^{t}$. Let us check that $\lambda=0$ is simple. Take $\chi=(f, g)^{t}$ such that $\mathcal{L}_{c}^{(2)} \chi=(0,0)^{t}$. Then we obtain that $B^{-1}(g)=0$ and $\mathcal{L}_{s n} f=0$. But we conclude that $f=\alpha q_{c, 1}^{\prime}$ and therefore $(f, g)=\alpha\left(q_{c, 1}^{\prime}, 0\right)$. Thus we have that $\lambda=0$ is simple. Now, given $\zeta=(f, g)^{t}$ we have that

$$
\left\langle\mathcal{L}_{c}^{(2)} \zeta, \zeta\right\rangle=\left\langle\mathcal{L}_{s n} f, f\right\rangle+\left\|B^{-1 / 2} g\right\|^{2},
$$

where $B^{-1 / 2}$ is the square root of the positive linear operator $B^{-1}$. So, by taking $\chi_{0}$ such that $\mathcal{L}_{s n} \chi_{0}=\lambda_{0} \chi_{0}$ for $\lambda_{0}<0$ and $\zeta_{0}=\left(\chi_{0}, 0\right)^{t}$, we obtain from (4.5) that $\left\langle\mathcal{L}_{c}^{(2)} \zeta_{0}, \zeta_{0}\right\rangle=\left\langle\mathcal{L}_{s n} \chi_{0}, \chi_{0}\right\rangle<0$. Therefore, $\mathcal{L}_{c}^{(2)}$ has a negative eigenvalue, which must be simple. Now, let $\zeta_{1}=\left(\chi_{0}, 0\right)^{t}$ and $\zeta_{2}=\left(q_{c, 1}^{\prime}, 0\right)^{t}$. Then for $\varphi=(f, g)^{t},\|\varphi\|=1$, it follows from the Min-max principle that the third eigenvalue for $\mathcal{L}_{c}^{(2)}, \eta_{3}$, satisfies

$$
\begin{aligned}
\eta_{3}=\sup _{\left[\xi_{1}, \xi_{2}\right]} \inf _{\varphi \perp \xi_{1}, \varphi \perp \xi_{2}}\left\langle\mathcal{L}_{c}^{(2)} \varphi, \varphi\right\rangle & \geqq \inf _{\varphi \perp \zeta_{1}, \varphi \perp \zeta_{2}}\left\langle\mathcal{L}_{c}^{(2)} \varphi, \varphi\right\rangle \\
& \geqq \inf _{f \perp \chi_{0}, f \perp q_{c, 1}^{\prime}}\left[\left\langle\mathcal{L}_{s n} f, f\right\rangle+\left\|B^{1 / 2} g\right\|^{2}\right] \geqq \delta_{0}>0,
\end{aligned}
$$

where in the last inequality we have used the spectral properties of linear operator $\mathcal{L}_{s n}$.

Finally, we are able to establish for $n=2$ the hypothesis (H2).

THEOREM 4.3. Suppose that $1<c<\sqrt{a / b}$. Then $\mathcal{L}_{c, 2}$ has exactly one negative simple eigenvalue with eigenfunction $\chi_{c} \in H_{\text {per }}^{1}[(0, L)] \times H_{\text {per }}^{1}([0, L])$, has its kernel spanned by $\partial_{x} \Psi_{c, 1} \in X$, and the rest of the spectrum in $H_{p e r}^{2}[(0, L)] \times H_{p e r}^{2}([0, L])$ is positive and away from zero.

Proof. From the spectral analysis of the operator $\mathcal{L}_{c}^{(2)}$ on $H_{p e r}^{2}[0, L] \times H_{\text {per }}^{2}([0, L])$ we have that $\mathcal{L}_{c, 2}$ has a unique negative simple eigenvalue. Now we will see that the kernel of $\mathcal{L}_{c, 2}$ is spanned by $\partial_{x} \Psi_{c, 1} \in X$. We know that $\left(q_{c}, p_{c}\right)^{t}$ satisfies the following equations:

$$
A q-c^{2} B q+\frac{4 c}{3} q^{3}=0, \quad p=\frac{1}{3} q^{3}-c B q .
$$

Then we also have that

$$
A q^{\prime}-c^{2} B q^{\prime}+4 c q^{2} q^{\prime}=0, \quad p^{\prime}=q^{2} q^{\prime}-c B q^{\prime} .
$$

Now, $\mathcal{L}_{c, 2}(f, g)^{t}=(0,0)^{t}$ is equivalent to

$$
B^{-1}\left(q^{2} f\right)-B^{-1}(g)=c f, \quad A f+2 c q^{2}+B^{-1}\left(q^{2} f\right) q^{2}-q^{2} B^{-1}(g)+c g=0 .
$$

Then $f$ satisfies $A f-c^{2} B f+4 c q^{2} f=0$. From (4.6) we conclude that $(f, g)=$ $\alpha\left(q_{c, 1}^{\prime}, p_{c, 1}^{\prime}\right)$. Again, from the spectral analysis on the operator $\mathcal{L}_{c}^{(2)}$, the rest of the spectrum of $\mathcal{L}_{c, 2}$ in $H_{p e r}^{2}([0, L]) \times H_{p e r}^{2}([0, L])$ is positive and away from zero. 
4.2. Concavity of $d$ for $c \in(1, \sqrt{a / b})$ and $n=2$. In order to establish orbital instability for such traveling wave solutions using Theorem (2.11) for $n=2$, we must verify that $d^{\prime \prime}(c)<0$, where $d$ is defined as

$$
d(c)=\mathcal{F}\left(\begin{array}{c}
q_{c, 1} \\
p_{c, 1}
\end{array}\right)
$$

where $p_{c, 1}=-c B q_{c, 1}+\frac{1}{3} q_{c, 1}^{3}$, with $q_{c, 1}$ being the wave solution obtained in Theorem 3.3. If we differentiate (4.7) with respect to $c$, we get that

$$
\begin{aligned}
d^{\prime}(c) & =\mathcal{N}\left(V_{c}\right) \\
& =\int_{0}^{L} p_{c, 1} q_{c, 1} d x \\
& =\int_{0}^{L}\left(-c q_{c, 1}^{2}-b c\left(q_{c, 1}^{\prime}\right)^{2}+\frac{1}{3} q_{c, 1}^{4}\right) d x .
\end{aligned}
$$

We will see that $d^{\prime}$ in (4.8) can be rewritten as

$$
d^{\prime}(c)=\frac{a+3 b c^{2}}{3\left(a-b c^{2}\right)} \int_{0}^{L} q_{c, 1}^{4}(x) d x-\frac{c(a-b)}{\left(a-b c^{2}\right)} \int_{0}^{L} q_{c, 1}^{2}(x) d x .
$$

In fact, multiplying equation $(3.1)$ by $q_{c}$ and integrating over $[0, L]$ we obtain that

$$
\left(a-b c^{2}\right) \int_{0}^{L}\left(q_{c, 1}^{\prime}\right)^{2} d x=\left(c^{2}-1\right) \int_{0}^{L} q_{c, 1}^{2}(x) d x-\frac{4 c}{3} \int_{0}^{L} q_{c, 1}^{4}(x) d x .
$$

So, replacing in $d^{\prime}(c)$ we obtain the desired expression. On the other hand, for $1<$ $c<\sqrt{a / b}$, we know from Theorem (3.3) that

$$
q_{c, 1}(x)=\frac{3 \eta_{1} \sqrt{c^{2}-1}}{2 c} \operatorname{sn}\left(\eta_{2} \sqrt{\frac{3\left(c^{2}-1\right)}{2 c\left(a-b c^{2}\right)}} x ; k_{1}\right),
$$

with $\eta_{1}^{2}(c)+\eta_{2}^{2}(c)=\frac{2 c}{3}$ and $k_{1}(c)=\frac{\eta_{1}(c)}{\eta_{2}(c)}$. Then a direct computation shows that

$$
\begin{aligned}
\int_{0}^{L} q_{c, 1}^{2}(x) d x & =\frac{6\left(a-b c^{2}\right) k_{1}^{2} K}{c L} \int_{0}^{4 K} \operatorname{sn}^{2}(y) d y \\
\int_{0}^{L} q_{c, 1}^{4}(x) d x & =\frac{144\left(a-b c^{2}\right)^{2} k_{1}^{4} K^{3}}{c^{2} L^{3}} \int_{0}^{4 K} \operatorname{sn}^{4}(y) d y \\
& =\frac{9\left(a-b c^{2}\right)\left(c^{2}-1\right) k_{1}^{4} K}{c^{2}\left(k_{1}^{2}+1\right) L} \int_{0}^{4 K} \operatorname{sn}^{4}(y) d y .
\end{aligned}
$$

But we know that $k_{1}^{2} \operatorname{sn}^{2}(y)=1-\operatorname{dn}^{2}(y)$, and that

$$
\begin{aligned}
& \int_{0}^{4 K} \operatorname{dn}^{2}(y) d y=4 \int_{0}^{K} \operatorname{dn}^{2}(y) d y=4 E \\
& \int_{0}^{4 K} \operatorname{dn}^{4}(y) d y=4 \int_{0}^{K} \operatorname{dn}^{4}(y) d y=\frac{4}{3}\left[\left(k_{1}^{2}-1\right) K+2\left(2-k_{1}^{2}\right) E\right],
\end{aligned}
$$


where $E$ and $F$ are the Elliptic integrals of the first class defined by

$$
E\left(k_{1}\right)=\int_{0}^{\pi / 2} \sqrt{1-k_{1}^{2} \sin ^{2} t} d t \text { and } F\left(k_{1}\right)=\int_{0}^{\pi / 2} \frac{1}{\sqrt{1-k_{1}^{2} \sin ^{2} t}} d t .
$$

As a consequence of this and previous formulas, we find that

$$
\begin{aligned}
\int_{0}^{L} q_{c, 1}^{2}(x) d x & =\frac{24\left(a-b c^{2}\right)}{c T_{0}} K(K-E) \\
\int_{0}^{L} q_{c, 1}^{4}(x) d x & =\frac{192\left(a-b c^{2}\right)^{2}}{c^{2} L^{3}} K^{3}\left[\left(k_{1}^{2}+2\right) K-2\left(k_{1}^{2}+1\right) E\right] \\
& =\frac{12\left(a-b c^{2}\right)\left(c^{2}-1\right)}{c^{2}\left(k_{1}^{2}+1\right) L} K\left[\left(k_{1}^{2}+2\right) K-2\left(k_{1}^{2}+1\right) E\right] .
\end{aligned}
$$

Replacing these into $d^{\prime}(c)$ we finally get that

$$
\begin{aligned}
d^{\prime}(c) & =\frac{4\left(a+3 b c^{2}\right)\left(c^{2}-1\right)}{c^{2} L} K\left[\left(\frac{k_{1}^{2}+2}{k_{1}^{2}+1}\right) K-2 E\right]-\frac{24(a-b)}{L} K[K-E] \\
& =\frac{4\left(a+3 b c^{2}\right)\left(c^{2}-1\right)}{c^{2} L} J_{1}(c)-\frac{24(a-b)}{L} J_{2}(c),
\end{aligned}
$$

where $J_{1}(c)=K\left[\left(\frac{k_{1}^{2}+2}{k_{1}^{2}+1}\right) K-2 E\right] \geq 0$ and $J_{2}(c)=K[K-E] \geq 0$.

The first observation is that $d$ is a non increasing function. In fact, a simple computation shows that

$$
2 J_{1}-3 J_{2}=\frac{\left(1-k_{1}^{2}\right) K^{2}-\left(1+k_{1}^{2}\right) K E}{1+k_{1}^{2}} \leq 0 .
$$

On the other hand,

$$
\begin{aligned}
d^{\prime}(c) & =\frac{4}{c^{2} L}\left(\left(a+3 b c^{2}\right)\left(c^{2}-1\right) J_{1}(c)-6(a-b) c^{2} J_{2}(c)\right) \\
& =\frac{4}{c^{2} L}\left(\left[\left(a+3 b c^{2}\right)\left(c^{2}-1\right)-4(a-b) c^{2}\right] J_{1}(c)+2(a-b) c^{2}\left(2 J_{1}-3 J_{2}(c)\right)\right) \\
& =\frac{4}{c^{2} L}\left(-\left(3 c^{2}+1\right)\left(a-b c^{2}\right) J_{1}(c)+2(a-b) c^{2}\left(2 J_{1}-3 J_{2}(c)\right)\right) \leq 0 .
\end{aligned}
$$

Now we have to compute $d^{\prime \prime}(c)$. We start computing $\frac{d k_{1}}{d c}$. To do this, we have to recall that $\eta$ is defined implicitly by the equation

$$
\Pi(\eta, c)=\frac{4 \sqrt{2} K\left(k_{1}(\eta)\right)}{\left(2 c / 3-\eta^{2}\right)^{1 / 2}}-\theta(c) L=\frac{4 \sqrt{2} K\left(k_{1}(\eta)\right)}{\left(2 c / 3-\eta^{2}\right)^{1 / 2}}-\sqrt{\frac{3\left(c^{2}-1\right)}{c\left(a-b c^{2}\right)}} L=0 .
$$

LEMMA 4.4.

$$
\frac{d}{d c} k_{1}(c)=\frac{k_{1}\left(1+k_{1}^{2}\right)\left(1-k_{1}^{2}\right) K\left(k_{1}\right) Q_{1}}{c H\left(k_{1}, c\right)} \geq 0
$$

where

$$
Q_{1}=\left(a-b c^{2}\right)\left(c^{2}-1\right) k_{1}^{2}+2(a-b) c^{2} \geq 0, \quad \text { and }
$$




$$
H\left(k_{1}, c\right)=2\left(a-b c^{2}\right)\left(c^{2}-1\right)\left[\left(1+k_{1}^{2}\right) E\left(k_{1}\right)-\left(1-k_{1}^{2}\right) K\left(k_{1}\right)\right] .
$$

Proof. First we have to observe that

$\partial_{\eta} k_{1}(\eta)=\frac{2 c \eta}{3 k_{1}\left(2 c / 3-\eta^{2}\right)^{2}}, 1+k_{1}^{2}=\frac{2 c}{3\left(2 c / 3-\eta^{2}\right)}, \quad$ and $\quad \frac{d}{d k_{1}} K\left(k_{1}\right)=\frac{E-\left(1-k_{1}^{2}\right) K}{k_{1}\left(1-k_{1}^{2}\right)}$.

Then we have that

$$
\begin{aligned}
\partial_{\eta} \Pi(\eta, c) & =\frac{4 \sqrt{2} \eta}{\left(2 c / 3-\eta^{2}\right)^{5 / 2}}\left[\left(2 c / 3-\eta^{2}\right) K+\frac{2 c K^{\prime}\left(k_{1}\right)}{3 k_{1}}\right] \\
& =\frac{4 \sqrt{2} k_{1}}{\left(2 c / 3-\eta^{2}\right)^{2}}\left[\left(\frac{2 c}{3}-\eta^{2}-\frac{2 c}{3 k_{1}^{2}}\right) K+\frac{2 c E\left(k_{1}\right)}{3 k_{1}^{2}\left(1-k_{1}^{2}\right)}\right] \\
& =\frac{4 \sqrt{2}(2 c / 3)}{\left(2 c / 3-\eta^{2}\right)^{2} k_{1}}\left[\frac{E\left(k_{1}\right)}{1-k_{1}^{2}}-\frac{K}{1+k_{1}^{2}}\right] \\
& =\frac{6 \sqrt{2}\left(1+k_{1}^{2}\right)}{c k_{1}\left(1-k_{1}^{2}\right)}\left[\left(1+k_{1}^{2}\right) E\left(k_{1}\right)-\left(1-k_{1}^{2}\right) K\right] .
\end{aligned}
$$

Moreover, it is straightforward to see that

$$
L \theta^{\prime}(c)=\frac{3\left(b c^{2}\left(c^{2}-1\right)+(a-b) c^{2}+a-b c^{2}\right) L}{2 \theta c^{2}\left(a-b c^{2}\right)^{2}}=\frac{\left(b c^{4}+(a-3 b) c^{2}+a\right) \theta L}{2 c\left(c^{2}-1\right)\left(a-b c^{2}\right)} \geq 0 .
$$

On the other hand,

$$
\begin{aligned}
\partial_{c} \Pi(\eta, c) & =-\frac{4 \sqrt{2} K\left(k_{1}\right)}{3\left(2 c / 3-\eta^{2}\right)^{3 / 2}}-\theta^{\prime}(c) T_{0} \\
& =-\frac{4 \sqrt{2} K\left(k_{1}\right)}{3\left(2 c / 3-\eta^{2}\right)^{3 / 2}}-\frac{\left(b c^{4}+(a-3 b) c^{2}+a\right)}{2 c\left(c^{2}-1\right)\left(a-b c^{2}\right)} \frac{4 \sqrt{2} K\left(k_{1}\right)}{\left(2 c / 3-\eta^{2}\right)^{1 / 2}} \\
& =-\frac{4 \sqrt{2} K\left(k_{1}\right)}{3\left(2 c / 3-\eta^{2}\right)^{3 / 2}}\left[1+\frac{3\left(b c^{4}+(a-3 b) c^{2}+a\right)}{2 c\left(a-b c^{2}\right)\left(c^{2}-1\right)}\left(2 c / 3-\eta^{2}\right)\right] \\
& =-\frac{4 \sqrt{2} K\left(k_{1}\right)}{3\left(2 c / 3-\eta^{2}\right)^{3 / 2}}\left[1+\frac{b c^{4}+(a-3 b) c^{2}+a}{\left(a-b c^{2}\right)\left(c^{2}-1\right)\left(1+k_{1}^{2}\right)}\right] \\
& =-\frac{2 \sqrt{2} K\left(k_{1}\right)\left(1+k_{1}^{2}\right) k_{1}}{c \eta}\left[\frac{\left(a-b c^{2}\right)\left(c^{2}-1\right) k_{1}^{2}+2(a-b) c^{2}}{\left(a-b c^{2}\right)\left(c^{2}-1\right)\left(1+k_{1}^{2}\right)}\right]
\end{aligned}
$$

Using this and chain rule, we have that

$$
\begin{aligned}
\frac{d}{d c} \eta(c) & =-\frac{\partial_{c} \Pi(\eta, c)}{\partial_{\eta} \Pi(\eta, c)} \\
& =\frac{k_{1}^{2}\left(1-k_{1}^{2}\right) K\left(k_{1}\right) Q_{1}}{3 \eta\left(a-b c^{2}\right)\left(c^{2}-1\right)\left(1+k_{1}^{2}\right)\left[\left(1+k_{1}^{2}\right) E\left(k_{1}\right)-\left(1-k_{1}^{2}\right) K\left(k_{1}\right)\right]} \geq 0,
\end{aligned}
$$

where

$$
Q_{1}=\left(a-b c^{2}\right)\left(c^{2}-1\right) k_{1}^{2}+2(a-b) c^{2} \geq 0 .
$$

As a consequence of this,

$$
\frac{d}{d c} k_{1}(c)=\partial_{\eta} k_{1}(\eta) \frac{d}{d c} \eta(c)
$$




$$
\begin{aligned}
& =\frac{2 c \eta}{3 k_{1}\left(2 c / 3-\eta^{2}\right)^{2}} \frac{k_{1}^{2}\left(1-k_{1}^{2}\right) K\left(k_{1}\right) Q_{1}}{3 \eta\left(a-b c^{2}\right)\left(c^{2}-1\right)\left(1+k_{1}^{2}\right)\left[\left(1+k_{1}^{2}\right) E\left(k_{1}\right)-\left(1-k_{1}^{2}\right) K\left(k_{1}\right)\right]} \\
& =\frac{k_{1}\left(1+k_{1}^{2}\right)\left(1-k_{1}^{2}\right) K\left(k_{1}\right) Q_{1}}{2 c\left(a-b c^{2}\right)\left(c^{2}-1\right)\left[\left(1+k_{1}^{2}\right) E\left(k_{1}\right)-\left(1-k_{1}^{2}\right) K\left(k_{1}\right)\right]} \geq 0 .
\end{aligned}
$$

Finally we are able to establish the concavity of $d$.

LEMma 4.5. There are $1<c_{*} \leq c^{*}<\sqrt{a / b}$ such that $d(c)$ is concave for $c \in\left(1, c_{*}\right)$ and for $c \in\left(c^{*}, \sqrt{a / b}\right)$.

Proof. We have that

$$
\frac{L c^{3} d^{\prime \prime}(c)}{4}=2\left(3 b c^{4}+a\right) J_{1}+\left(a+3 b c^{2}\right)\left(c^{2}-1\right)\left(c J_{1}^{\prime}(c)\right)-6(a-b) c^{2}\left(c J_{2}^{\prime}(c)\right),
$$

where $^{\prime}=\frac{d}{d c}$. Now we observe that

$$
\begin{aligned}
\left(a+3 b c^{2}\right)\left(c^{2}-1\right) c J_{1}^{\prime}(c)-6(a-b) c^{2}\left(c J_{2}^{\prime}(c)\right) & =\frac{2 K Q_{1}\left(H_{1} K E+H_{2} K^{2}+H_{3} E^{2}\right)}{\left(1+k_{1}^{2}\right) H\left(k_{1}, c\right)} \\
& =\frac{K W}{\left(1+k_{1}^{2}\right) H\left(k_{1}, c\right)}
\end{aligned}
$$

where

$$
\begin{aligned}
& W=2 Q_{1}\left(H_{1} K E+H_{2} K^{2}+H_{3} E^{2}\right), \\
& H_{1}=\left(a+3 b c^{2}\right)\left(c^{2}-1\right)\left(2+k_{1}^{2}\right)\left(1+k_{1}^{2}\right)-6(a-b) c^{2}\left(1+k_{1}^{2}\right)^{2}, \\
& H_{2}=-\left(a+3 b c^{2}\right)\left(c^{2}-1\right)\left(1+2 k_{1}^{2}\right)\left(1-k_{1}^{2}\right)+3(a-b) c^{2}\left(1+k_{1}^{2}\right)^{2}\left(1-k_{1}^{2}\right), \\
& H_{3}=\left[-\left(a+3 b c^{2}\right)\left(c^{2}-1\right)+3(a-b) c^{2}\right]\left(1+k_{1}^{2}\right)^{2} .
\end{aligned}
$$

By defining $\Gamma=\left(a+3 b c^{2}\right)\left(c^{2}-1\right), \Theta=3(a-b) c^{2}$, and

$$
\begin{array}{ll}
\gamma_{1}=\left(2+k_{1}^{2}\right)\left(1+k_{1}^{2}\right), & \gamma_{2}=\left(1+2 k_{1}^{2}\right)\left(1-k_{1}^{2}\right), \gamma_{3}=\left(1+k_{1}^{2}\right)^{2}, \\
\eta_{1}=2\left(1+k_{1}^{2}\right)^{2}, & \eta_{2}=\left(1+k_{1}^{2}\right)^{2}\left(1-k_{1}^{2}\right), \quad \eta_{3}=\left(1+k_{1}^{2}\right)^{2},
\end{array}
$$

we find that

$$
\begin{aligned}
& H_{1}=\Gamma \gamma_{1}-\Theta \eta_{1}, \\
& H_{2}=-\Gamma \gamma_{2}+\Theta \eta_{2}, \\
& H_{3}=-\Gamma \gamma_{3}+\Theta \eta_{3} .
\end{aligned}
$$

Moreover, we also have that

$$
\begin{aligned}
& H_{1}=\left(\Gamma-\frac{4}{3} \Theta\right) \gamma_{1}+\Theta\left(\frac{4}{3} \gamma_{1}-\eta_{1}\right), \\
& H_{2}=-\left(\Gamma-\frac{4}{3} \Theta\right) \gamma_{2}+\Theta\left(\eta_{2}-\frac{4}{3} \gamma_{2}\right), \\
& H_{3}=-\left(\Gamma-\frac{4}{3} \Theta\right) \gamma_{3}+\Theta\left(\eta_{3}-\frac{4}{3} \gamma_{3}\right) .
\end{aligned}
$$


In particular,

$$
\begin{aligned}
H_{1} K E+H_{2} K^{2}+H_{3} E^{2} & =\left(\Gamma-\frac{4}{3} \Theta\right)\left(\gamma_{1} K E-\gamma_{2} K^{2}-\gamma_{3} E^{2}\right) \\
& +\frac{\Theta}{3}\left(2\left(1-k_{1}^{4}\right) K E-\left(1-k_{1}^{2}\right)^{2}\left(1+3 k_{1}^{2}\right) K^{2}-\left(1+k_{1}^{2}\right)^{2} E^{2}\right) .
\end{aligned}
$$

Now, using that $1+k_{1}^{2} \geq\left(1+2 k_{1}^{2}\right)\left(1-k_{1}^{2}\right)$ we are able to see that

$$
\begin{aligned}
\gamma_{1} K E-\gamma_{2} K^{2}-\gamma_{3} E^{2} & =\left(2+k_{1}^{2}\right)\left(1+k_{1}^{2}\right) K E-\left(1+2 k_{1}^{2}\right)\left(1-k_{1}^{2}\right) K^{2}-\left(1+k_{1}^{2}\right)^{2} E^{2} \\
& =K\left[\left(1+k_{1}^{2}\right) E-\left(1+2 k_{1}^{2}\right)\left(1-k_{1}^{2}\right) K\right]+\left(1+k_{1}^{2}\right)^{2}[K-E] E \\
& \geq\left(\left(1+2 k_{1}^{2}\right)\left(1-k_{1}^{2}\right) K[E-K]+\left(1+k_{1}^{2}\right)^{2}[K-E] E\right) \\
& \geq[K-E]\left(\left(1+k_{1}^{2}\right)^{2} E-\left(1+2 k_{1}^{2}\right)\left(1-k_{1}^{2}\right) K\right) \\
& \geq 0,
\end{aligned}
$$

since we have that $K-E>0$ and $\left(1+k_{1}^{2}\right)^{2} E-\left(1+2 k_{1}^{2}\right)\left(1-k_{1}^{2}\right) K \geq 0$.

Note that we also have for $c \in(1, \sqrt{a / b})$ that

$$
\Gamma-\frac{4}{3} \Theta=\left(a+3 b c^{2}\right)\left(c^{2}-1\right)-4(a-b) c^{2}=-\left(3 c^{2}+1\right)\left(a-b c^{2}\right) \leq 0,
$$

which implies that for $c \in(1, \sqrt{a / b})$ and $k_{1} \in(0,1)$,

$$
\left(\Gamma-\frac{4}{3} \Theta\right)\left(\gamma_{1} K E-\gamma_{2} K^{2}-\gamma_{3} E^{2}\right) \leq 0
$$

It can be also shown using MATLAB that

$$
G\left(k_{1}\right)=2\left(1-k_{1}^{4}\right) K E-\left(1-k_{1}^{2}\right)^{2}\left(1+3 k_{1}^{2}\right) K^{2}-\left(1+k_{1}^{2}\right)^{2} E^{2} \leq 0, \text { for } k_{1} \in(0,1) .
$$

In figure (4.1) we display the coefficient $G\left(k_{1}\right)$ as a function of the modulus $k_{1}$. In this plot we can see clearly that $G\left(k_{1}\right) \leq 0$ for $0 \leq k_{1} \leq 1$.

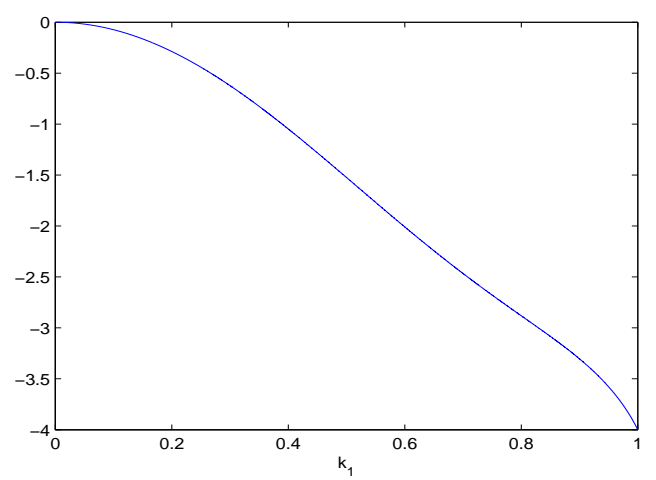

FiguRE 4.1. The coefficient $G\left(k_{1}\right)$ as a function of the modulus $k_{1}$.

As a consequence, we have established that

$$
W=2 Q_{1}\left(H_{1} K E+H_{2} K^{2}+H_{3} E^{2}\right)<0, \text { for } c \in(1, \sqrt{a / b}), \quad k_{1} \in(0,1) .
$$


By using (4.17) and (4.19), we see that $d^{\prime \prime}$ can be written as

$$
\frac{L c^{3} d^{\prime \prime}(c)}{4}=\frac{K\left\{2\left(3 b c^{4}+a\right)\left(a-b c^{2}\right)\left(c^{2}-1\right)\left[\left(2+k_{1}^{2}\right) K-2\left(1+k_{1}^{2}\right) E\right]+W_{1}\right\}}{\left(1+k_{1}^{2}\right)\left(a-b c^{2}\right)\left(c^{2}-1\right)},
$$

where

$$
W_{1}=\frac{W}{2\left(1+k_{1}^{2}\right) E\left(k_{1}\right)-\left(1-k_{1}^{2}\right) K\left(k_{1}\right)} .
$$

But from (4.15), we observe for either $\beta=1^{+}$or $\beta=(\sqrt{a / b})^{-}$that

$$
\begin{aligned}
& \lim _{c \rightarrow \beta}\left(a-b c^{2}\right)\left(c^{2}-1\right)\left[\left(2+k_{1}^{2}\right) K\left(k_{1}\right)-2\left(1+k_{1}^{2}\right) E\left(k_{1}\right)\right] \\
\leq & \lim _{c \rightarrow \beta} 4\left(1+k_{1}^{2}\right)\left(a-b c^{2}\right)\left(c^{2}-1\right) K\left(k_{1}\right) \leq 8 \lim _{c \rightarrow \beta}\left(a-b c^{2}\right)\left(c^{2}-1\right) K\left(k_{1}\right)=0 .
\end{aligned}
$$

Moreover, there exists $0<M<\infty$ such that

$$
\sup _{k_{1} \in[0,1]}\left[\left(1+k_{1}^{2}\right) E\left(k_{1}\right)-\left(1-k_{1}^{2}\right) K\left(k_{1}\right)\right]=M .
$$

As a consequence of these facts, we conclude that there are $1<c_{*} \leq c^{*}<\sqrt{a / b}$ such that $d$ is concave, since

$$
d^{\prime \prime}(c)=\frac{4 K\left\{2\left(3 b c^{4}+a\right)\left(a-b c^{2}\right)\left(c^{2}-1\right)\left[\left(2+k_{1}^{2}\right) K-2\left(1+k_{1}^{2}\right) E\right]+W_{1}\right\}}{L c^{3}\left(1+k_{1}^{2}\right)\left(a-b c^{2}\right)\left(c^{2}-1\right)} \leq 0
$$

in that range, since $2\left(1+k_{1}^{2}\right) E\left(k_{1}\right)-\left(1-k_{1}^{2}\right) K\left(k_{1}\right)>0$.

REMARK 4.6. As we have seen in previous computations, to determine analytically the sign of $d^{\prime \prime}$ is not quite trivial. Note that the relation between $k_{1}$ and $c$ is not explicit, and so, we do not know where the range of $k_{1}$ is localized. In accordance with numerical simulations for many values of the parameter $a$ and $b$, we conjecture that $d$ is in fact concave in $(1, \sqrt{a / b})$. To give an insight on this conjecture, we performed some numerical experiments. See numerical Section 5 below.

As a consequence of the previous result and the general instability result (2.11), we obtain for $n=2$ the following instability result.

Corollary 4.7. Let $a>b>\frac{a}{9}, 1<c<\min \left(c_{*}, \sqrt{\frac{1+\frac{4 \pi^{2} a}{L^{2}}}{1+\frac{4 \pi^{2} b}{L^{2}}}}\right), n=2$, and let $q_{c, 1}$ be the snoidal wave branch of period $L$ given in Theorem 3.3. Then the orbit $\left\{\left(q_{c, 1}(\cdot+\right.\right.$ $\left.\left.s), p_{c, 1}(\cdot+s)\right)\right\}_{s \in \mathbb{R}}$ is $X$-unstable with regards to periodic perturbations and the flow generated by system (2.3).

\section{Description of numerical experiments}

For solving the Cauchy problem associated to the generalized system

$$
\begin{aligned}
& q_{t}=r_{x}, \\
& p_{t}=\partial_{x}\left(A q-r q^{n}\right),
\end{aligned}
$$

we use a spectral numerical scheme as in [17], where we use that $p=B r+\frac{1}{n+1} q^{n+1}$. In previous sections we have explained the method to build spatially-periodic traveling wave solutions to system (1.1) when $n=2$ in two different cases, depending on the 

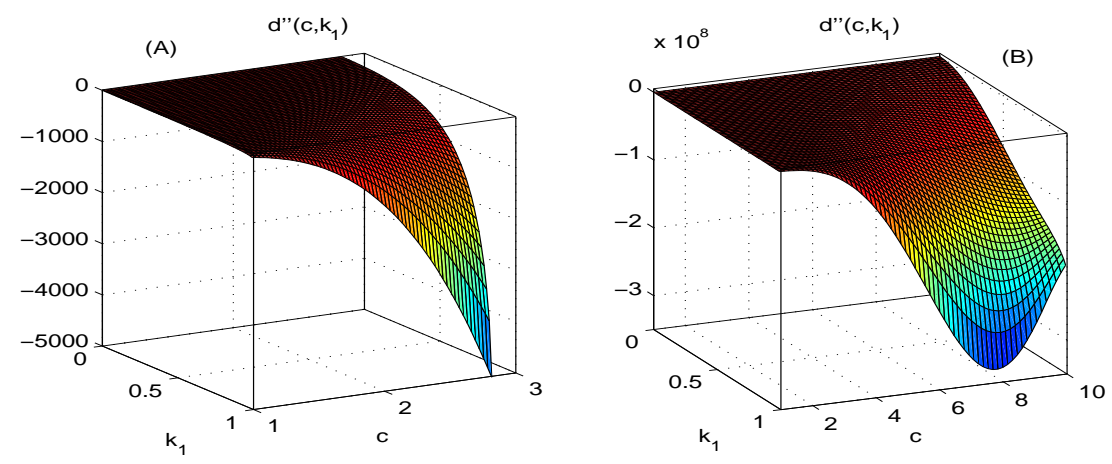

Figure 5.1. The coefficient $d^{\prime \prime}\left(c, k_{1}\right)$ for two values of the model's parameters a and $b$. In $(A)$ $a=0.8, b=0.1$. In $(B) a=50, b=0.5$. Observe that $d^{\prime \prime}\left(c, k_{1}\right)$ is negative in the entire range.
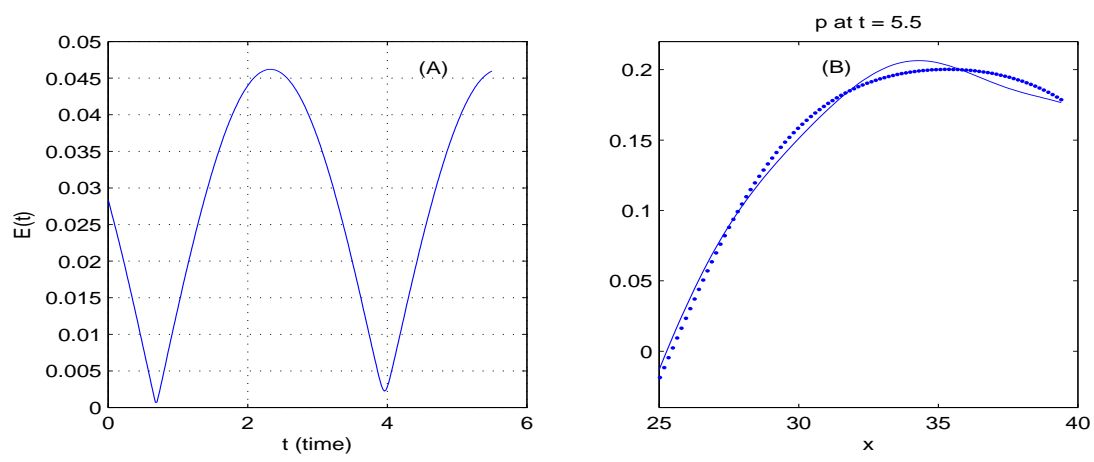

Figure 5.2. (A) Deviation $E(t)$ of an unstable snoidal wave solution with $a=0.8, b=0.1$. The perturbation has period $L=39.56$ (fundamental period of the snoidal wave) and wave speed $c=1.03$. (B) Comparison between the perturbed numerical solution and the analytic snoidal solution.

regime of wave velocity and the parameters $a$ and $b$. For $c \in(1, \sqrt{a / b})$, we proved analytically the instability of the $L$-spatially periodic snoidal wave solution $\left(q_{c, 1}, p_{c, 1}\right)$ under perturbations having the same period $L$, provided that the wave speed $c$ is near enough the critical value 1 and $9 b>a>b$.

As mentioned above, it is difficult to study analytically the sign of the function $d^{\prime \prime}(c)$ on the entire range $(1, \sqrt{a / b})$. A large set of numerical experiments performed with MATLAB plotting the coefficient $d^{\prime \prime}(c)$ for several values of the parameters $a$ and $b$ indicates that $d^{\prime \prime}(c)$ is negative for all $c \in(1, \sqrt{a / b})$. To illustrate this issue, we display in figure (5.1) the coefficient $d^{\prime \prime}\left(c, k_{1}\right)$ as a function of the wave speed $0<c<\sqrt{a / b}$ and the modulus $k_{1} \in(0,1)$, for $a=0.8, b=0.1$ and for $a=50, b=0.5$.

Even though we know that we can not apply the Grillakys-Shatah-Strauss approach to study stability/instability of cnoidal wave solutions in the case $c \in(\sqrt{a / b}, 1)$ (cnoidal type solutions) we will provide numerical evidence of instability in such a case in Section 5.2 , by using the numerical scheme implemented. 

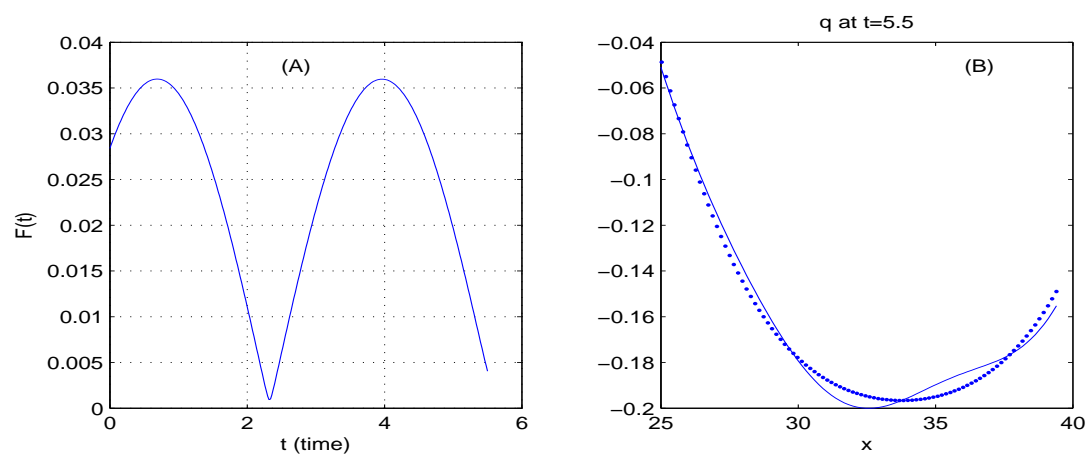

Figure 5.3. (A) Deviation $F(t)$ of an unstable snoidal wave solution with $a=0.8, b=0.1$. The perturbation has period $L=39.56$ (fundamental period of the scnoidal wave) and wave speed $c=1.03$. Comparison between the perturbed numerical solution and the analytic snoidal solution.
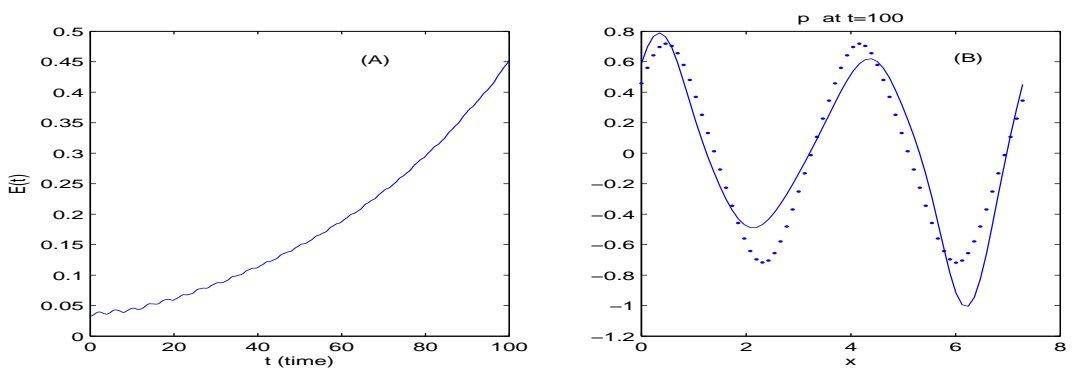

Figure 5.4. (A) Deviation $E(t)$ of an unstable cnoidal wave solution with $a=0.3, b=0.7$. The perturbation has period $2 L$, with fundamental period $L=3.69886$ and the wave speed $c=0.8$.
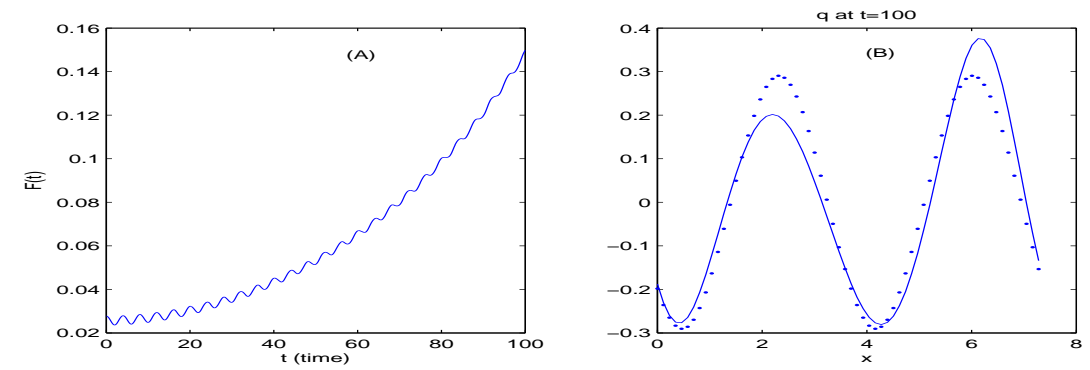

FiguRE 5.5. (A) Deviation $F(t)$ of an unstable cnoidal wave solution with $a=0.3, b=0.7$. The perturbation has period $2 L$, with fundamental $L=3.69886$ and the wave speed $c=0.8$.

5.1. Instability of snoidal waves. To measure the deviation of the solution pair $(q(t), p(t))$ from the orbit of the snoidal wave $q_{c}=q_{c, 1}, p_{c}=p_{c, 1}$, we introduce the time-dependent functions 


$$
\begin{aligned}
E(t) & =\min _{k=1, . . N} \sqrt{\frac{L}{N} \sum_{j=1}^{N}\left[\left|p(j \Delta x, t)-p_{c}(j \Delta x+k \Delta x)\right|^{2}+\left|p_{x}(j \Delta x, t)-p_{c}^{\prime}(j \Delta x+k \Delta x)\right|^{2}\right]},
\end{aligned}
$$

$$
F(t)=\min _{k=1, . . N} \sqrt{\frac{L}{N} \sum_{j=1}^{N}\left[\left|q(j \Delta x, t)-q_{c}(j \Delta x+k \Delta x)\right|^{2}+\left|q_{x}(j \Delta x, t)-q_{c}^{\prime}(j \Delta x+k \Delta x)\right|^{2}\right]}
$$

where $N$ is the number of grid points used in the numerical calculation of $p(x, t)$ and $q(x, t)$ on the computational domain $[0, L]$. Furthermore, $p_{x}, p_{c}^{\prime}, q_{x}, q_{c}^{\prime}$ denote the derivatives with respect to $x$ of the functions $p, p_{c}, q, q_{c}$, respectively. In the numerical experiments to be presented, we will study the behavior of the deviations $E(t)$ and $F(t)$ as long as time $t$ increases. These functions are computed at every time step by using the numerical solution $(q(t), p(t))$ to system (5.1)-(5.2).

We explore the dynamics of the branch of snoidal wave solution $q_{c}=q_{c, 1}, p_{c}=p_{c, 1}$ for (5.1)-(5.2) when time increases. We run the numerical scheme with perturbed initial conditions

$$
\begin{aligned}
& q(x, 0)=q_{c}(x)+\epsilon \cos \left(5 \frac{2 \pi x}{L}\right), \\
& p(x, 0)=p_{c}(x)+\epsilon \sin \left(5 \frac{2 \pi x}{L}\right),
\end{aligned}
$$

where the scale $\epsilon>0$ controls the strength of the perturbation introduced into the cnoidal wave $\left(q_{c}, p_{c}\right)$. Observe that this amounts to introducing perturbations having the same period $L$ that the snoidal wave. In our first simulation we set $\epsilon=0.005$. Furthermore, the initial deviations introduced are $E(0)=0.0284, F(0)=0.0285$. We take $a=0.8, b=0.1, \eta_{1}=0.5472, \eta_{2}=0.6223$, the wave speed adopted is $c=1.03$ (i.e. near one), and the fundamental period of the snoidal wave is $L=39.56$. This set of parameters satisfies all conditions required in Section 3. The numerical parameters are $2^{8}$ FFT points equally spaced along the computational domain $[0, L]$ and the time step is $\Delta t=0.001$. In figures $(5.2)(\mathrm{A})$ and $(5.3)(\mathrm{A})$ we present the outcome of the deviations $E(t)$ and $F(t)$ for the solutions $p(x, t)$ and $q(x, t)$ of the perturbed problem. Observe that the total deviation $E(t)+F(t)$ increases up to approximately 1.5 times the initial perturbation introduced, which indicates an unstable nature of the snoidal wave solution. This is in accordance with the analytic results presented above for $c$ near one. In figures (5.2)(B) and (5.3)(B) we can see the profiles corresponding to the numerical solution to system (5.1)-(5.2) (with perturbed initial data in the form (5.5)-(5.6)), and the snoidal wave $\left(q_{c, 1}, p_{c, 1}\right)$ after an appropriate spatial translation in order to compare their shapes. Observe that the discrepancy of the derivative of these profiles is very dramatic near the left-side boundary and at the center of the interval.

5.2. Instability of cnoidal waves. By using the implemented numerical scheme we provide experiments showing that the cnoidal-wave solution $q_{c}=q_{c, 2}, p_{c}=$ 
$p_{c, 2}$ with period $L$ is unstable when perturbed by $2 L$-periodic disturbances of the form

$$
\begin{gathered}
q(x, 0)=q_{c}(x)+\epsilon q_{c}(x / 2), \\
p(x, 0)=p_{c}(x)+\epsilon p_{c}(x / 2),
\end{gathered}
$$

with $\epsilon=0.05$. In this case, we do not have analytic results since it is not possible to adapt the Grillakis-Shatah-Strauss approach.

$$
\begin{aligned}
& E(t)=\min _{k=1, . . N} \sqrt{\frac{L}{N} \sum_{j=1}^{N}\left|p(j \Delta x, t)-p_{c}(j \Delta x+k \Delta x)\right|^{2}}, \\
& F(t)=\min _{k=1, . . N} \sqrt{\frac{L}{N} \sum_{j=1}^{N}\left|q(j \Delta x, t)-q_{c}(j \Delta x+k \Delta x)\right|^{2}} .
\end{aligned}
$$

Here we set $a=0.3, b=0.7, \eta_{1}=0.2582, \eta_{2}=0.7746$, the wave speed $c=0.8$, and the fundamental period $L=3.69886$. The numerical parameters are $2^{6} \mathrm{FFT}$ points equally distributed on the computational domain $[0,2 L]$ and the time step is $\Delta t=3.3 \times 10^{-4}$. In figures $(5.4)(\mathrm{A}),(5.5)(\mathrm{A})$ we feature the deviations $E(t)$ and $F(t)$ computed in this numerical simulation. The initial deviations are $E(0)=3.28 \times 10^{-2}$ and $F(0)=$ $2.77 \times 10^{-2}$. In figures (5.4)(B) and (5.5)(B) we can see the profiles corresponding to the numerical solution to system (5.1)-(5.2) (with initial data (5.7)-(5.8)), and the cnoidal wave $\left(q_{c, 2}, p_{c, 2}\right)$ after an appropriate spatial translation in order to compare their shapes. The deviation of the perturbed solution increases up to roughly 10 times its initial deviation, providing us with clear numerical evidence with regard to the instability of this type of periodic solutions under $2 L$-periodic disturbances.

Acknowledgments. This work was supported by Universidad del Valle, CaliColombia.

\section{REFERENCES}

[1] E. Alarcon, J. Angulo, and J.F. Montenegro, Stability and instability of solitary waves for a nonlinear dispersive system, Nonlin. Anal., 36, 1015-1035, 1999.

[2] J.P. Albert, J. Bona, and D.B. Henry, Sufficient conditions for stability of solitary-wave solutions of model equations for long waves, Physica D, 343-366, 1987.

[3] J. Angulo, Nonlinear stability of periodic travelling wave solutions to the Schrödinger and the modified Korteweg-de Vries equations, J. Diff. Eqs., 235, 1-30, 2007.

[4] J. Angulo, J. Bona, and M. Scialom, Stability of cnoidal waves, Adv. Diff. Eqs., 11, 1321-1374, 2006.

[5] D. Benney, and J. Luke, Interactions of permanent waves of finite amplitude, J. Math. Phys., 43, 309-313, 1964.

[6] J. Bona, P. Souganidis, and W. Strauss, Stability and instability of solitary waves of Kortewegde Vries type, Proc. R. Soc. London A, 411(1841), 395-412, 1987.

[7] P. Byrd and M. Friedman, Handbook of Elliptic Integrals for Engineers and Scientist, Spriger Verlag. N.Y, 1971.

[8] T. Gallay and M. Hârâgus, Orbital stability of periodic waves for the nonlinear Schrödinger equation, J. Dyn. Diff. Equ., 19(4), 825-865, 2007.

[9] M. Grillakis, J. Shatah, and W. Strauss, Stability theory of solitary waves in presence of symmetry I, Func. Anal., 74, 160-197, 1987.

[10] E. Ince, The periodic Lamé function, Proc. Roy. Soc., 60, 47-63, 1940.

[11] Y. Kivshar and G. Agrawal, Optical Solitons: From Fibers to Photonic Crystals, Academic Press, 1 (Ed.), 2003.

[12] W. Magnus and S. Winkler, Hill's Equation, Tracts in Pure and Appl. Math., Wesley. N.Y, 20, 1976. 
[13] P. Milewski, A formulation for water waves over topography, Stud. Appl. Math., 100, 95-106, 1998.

[14] P. Milewski and E. Tabak, A pseudospectral procedure for the solution of nonlinear wave equations with examples from free-surface flows, SIAM J. Sci. Comput., 21(3), 1102-1114, 1999.

[15] P. Milewski and K. Berger, The generation and evolution of lump solitary waves in surfacetension-dominated flows, SIAM J. Appl. Math., 61(3), 731-750, 2000.

[16] P. Milewski and J.B. Keller, Three dimensional water waves, Stud. Appl. Math., 37, 149-166, 1996.

[17] J. Muñoz, Instability and long-time evolution of cnoidal wave solutions for a Benney-Luke equation, Int. J. Non-linear Mech., 44, 999-1010, 2009.

[18] D. Pelinovsky, Inertia law for spectral stability of solitary waves in coupled nonlinear Schrödinger equations, Proc. Roy. Soc. A, 461, 783-812, 2005.

[19] J. Quintero and R. Pego, Two-Dimensional solitary waves for a Benney-Luke equation, Physica D, 132, 476-496, 1999.

[20] J. Quintero, A note on the stability and instability of travelling wave of Korteweg-de Vries Type: The periodic case, Revista de Ciencias Universidad del Valle, 1-2, 69-86, 2010.

[21] J. Quintero and J. Angulo, Existence and Orbital Stability of Cnoidal Waves for a 1D Boussinesq Equation, Int. J. Math. and Math. Sci., Article ID 52020, 2007.

[22] P. Shukla, I. Kourakis, B. Eliasson, M. Marklund, and L. Stenflo, Instability and evolution of nonlinearly interacting water waves, Phys. Rev. Lett., 97, 2006.

[23] B. Tan and J. Boyd, Stability and long time evolution of the periodic solutions to the two coupled nonlinear Schrödinger equations, Chaos, Solitons and Fractals, 12, 721-734, 2001.

[24] N.J. Zabusky, A Synergetic Approach to Problems of Nonlinear Dispersive Wave Propagation and Interaction, Proceddings of the Symposium on Nonlinear Partial Differential Equations, W. Ames (Ed.), Academic Press Inc., New York, 223, 1967.

[25] H. Washimi and T. Taniuti, Propagation of ion-acoustic solitary waves of small amplitude, Phys. Rev. Lett., 17, 996-998, 1966.

[26] A.M. Wazwaz, Analytic study on the generalized fith-order KdV equation: New solitons and periodic solutions, Commun. Non. Sci. and Num. Sim., 12, 1172-1180, 2007.

[27] J. Bronski and M. Johnson, The modulational instability for a generalized KdV equation, Arch. Rat. Mech. Anal., 197(2), 357-400, 2010.

[28] M. Chen, C. Curtis, B. Deconinck, C. Lee, and N. Nguyen, Spectral stability of stationary solutions of a Boussinesq system describing long waves in dispersive media, SIAM J. Appl. Dyn. Sys., 9(3), 999-1018, 2010.

[29] B. Deconinck and M. Nivala, The stability analysis of the periodic traveling wave solutions of the MKdV equation, Stud. Appl. Math., 126, 17-48, 2010.

[30] M. Haragus and T. Kapitula, On the spectra of periodic waves for infinite-dimensional Hamiltonian systems, Physica D, 237(20), 2649-2671, 2008.

[31] M. Johnson, Nonlinear stability of periodic traveling wave solutions of the generalized Korteweg-de Vries equation, SIAM J. Math. Anal., 41(5), 1921-947, 2009.

[32] T. Kapitula, P. Kevrekidis, and B. Sandstede, Counting eigenvalues via the Krein signature in infinitedimensional Hamiltonian systems, Physica D, 195(3-4), 263-282, 2004.

[33] T. Kapitula, P. Kevrekidis, and B. Sandstede, Addendum: Counting eigenvalues via the Krein signature in infinite-dimensional Hamiltonian systems, Physica D, 201(1\&2), 199-201, 2005. 